OPEN ACCESS

Edited by:

Yuheng Luo,

Sichuan Agricultural University, China

Reviewed by:

Jing Zhang,

Shanghai Jiao Tong University, China

Christine Ann Butts,

The New Zealand Institute for Plant and Food Research Limited,

New Zealand

*Correspondence:

Xin'e Shi

xineshi@nwafu.edu.cn

Specialty section: This article was submitted to

Food Microbiology,

a section of the journal

Frontiers in Microbiology

Received: 05 March 2019

Accepted: 28 October 2019

Published: 20 November 2019

Citation:

Lu J, Zhang X, Liu Y, Cao H, Han Q, Xie B, Fan L, Li X, Hu J,

Yang $G$ and Shi X (2019) Effect of Fermented Corn-Soybean Meal on

Serum Immunity, the Expression of Genes Related to Gut Immunity, Gut Microbiota, and Bacterial Metabolites

in Grower-Finisher Pigs.

Front. Microbiol. 10:2620.

doi: 10.3389/fmicb.2019.02620

\section{Effect of Fermented Corn-Soybean Meal on Serum Immunity, the Expression of Genes Related to Gut Immunity, Gut Microbiota, and Bacterial Metabolites in Grower-Finisher Pigs}

Junfeng Lu, Xiaoyu Zhang, Yihao Liu, Haigang Cao, Qichun Han, Baocai Xie, Lujie Fan, Xiao Li, Jianhong Hu, Gongshe Yang and Xin'e Shi*

Key Laboratory of Animal Genetics, Breeding and Reproduction of Shaanxi Province, Laboratory of Animal Fat Deposition and Muscle Development, College of Animal Science and Technology, Northwest A\&F University, Yangling, China

Fermented corn-soybean meal (fermented feed, FF) is commonly used in swine production, but the effects of FF on gut health remain unclear. In this study, serum immunity, mRNA abundances of antimicrobial peptides (AMPs) and Toll-like receptors (TLR1-9), bacterial abundance in the duodenum and colon, and colonic metabolic phenotypes were determined in crossbred barrows (Duroc $\times$ Landrace $\times$ Large White) fed FF or normal feed (unfermented feed, UF) $(n=6)$. When compared to the UF group, the results showed that serum levels of $\lg G$ and $\operatorname{lgM}$ were significantly increased in FF group pigs $(P<0.05)$. FF significantly decreased the abundances of Bacteroides and Verrucomicrobia in the duodenum and decreased the abundances of Bacteroides, Proteobacteria, and Verrucomicrobia in the colon while it significantly increased the abundances of Firmicutes and Actinobacteria $(P<0.05)$. Furthermore, a Spearman's correlation analysis showed that serum immunity and the expression of genes related to gut immunity were associated with bacterial strains at the family level. Moreover, differentially abundant colonic microbiota were associated with colonic metabolites. LC-MS data analyses identified a total of 1,351 metabolites that markedly differed between the UF and FF groups. C5-Branched dibasic acid metabolism was significantly upregulated whereas the purine metabolism was significantly downregulated $(P<0.05)$ in the colonic digesta of pigs in the FF meal group compared to the UF meal group. Collectively, these results indicated that FF meal could influence serum immunity and the expression of genes related to gut immunity, correlating with the gut microbiota and bacterial metabolites in grower-finisher pigs. This study may provide an alternative strategy for improving the intestinal health of grower-finisher pigs.

Keywords: fermented feed, corn-soybean meal, immunity, microbiota, metabolite, pig 


\section{INTRODUCTION}

Antibiotics have long been used in swine production to maintain health and productivity. In recent years, the overuse of antibiotics in swine production has become an important concern (van der Fels-Klerx et al., 2011) and has led to the development of alternatives to antibiotics in feeds (Dibner and Richards, 2005), such as probiotics, prebiotics, and organic acids, for use in swine husbandry (Thacker, 2013; Markowiak and Slizewska, 2018). The microbial fermentation of feed has been proposed as a potential alternatives (Verstegen and Williams, 2002).

Extensive evidence has shown that microbial fermentation can improve the nutritional quality of pig feed by increasing the bioavailability of nutrients (Borling Welin et al., 2015; Jeong et al., 2016) and reducing the content of anti-nutritional factors (Missotten et al., 2015; Shi et al., 2017; Wang et al., 2018). Moreover, the use of fermented corn-soybean meal has been shown to improve the immune function of pigs (Zhu et al., 2017), as the consumption of FF has a positive impact on the gut microbiota and can improve the fecal microbial count and intestinal morphology in grower-finisher pigs (Dowarah et al., 2017). However, so far, information on the effects of FF on the health of pigs is limited.

Many studies have highlighted the correlation between gut microbiota and intestinal morphology, regulation of immunity, digestion of carbohydrates, and body health in livestock (Richards et al., 2005; Stanley et al., 2016; Gu et al., 2019; Guevarra et al., 2019). Recent evidence suggests that the gut microbiota plays a crucial role in livestock health and disease (Tremaroli and Backhed, 2012). A growing number of studies indicate that the homeostasis and composition of the gut microbiota is dynamically formed by many factors (Ananthakrishnan, 2015; Zhao et al., 2015), including age, time, feed, and probiotics (Isaacson and Kim, 2012). For instance, in grower-finisher pigs, fermented Mao-tai lees modulate the gut microbiota and increase the abundance of the potentially beneficial bacteria Lactobacillus and Akkermansia (Wang J. et al., 2019). Moreover, $45 \%$ feed of fish meal can be replaced by fermented soybean meal without negative effects on growth performance and intestinal integrity of juvenile large yellow croaker (Wang P. et al., 2019).

It remains uncertain whether FF boosts immunity and the composition of the gut microbiota of swine. Therefore, the aim of the present study was to assess the effects of corn-soybean FF on serum immunity factors and the expression of genes related to gut immunity. The results of an intestinal microbiota analysis indicated that corn-soybean FF significantly changed the composition of the gut microbiota and that metabolites significantly differed between pigs fed FF meal versus UF meal.

\section{MATERIALS AND METHODS}

\section{Ethics Statement}

This study was carried out in accordance with the recommendations of the Animal Welfare Committee of Northwest A\&F University (Yangling, Shaanxi Province, China).
The protocol was approved by the Animal Welfare Committee of Northwest A\&F University.

\section{Preparation and Composition of FF}

Corn-soybean meal was purchased from Beijing Dabeinong Technology Group Co., Ltd. (Beijing, China). Effective Microorganisms ${ }^{\mathrm{TM}}$ used in the present study are a mixture containing 60\% Lactobacillus, 20\% Clostridium, and 8\% Bifidobacteria and were purchased from Nongfukang Biological Technology Co., Ltd. (Zhengzhou, China) and diluted 1:30 $(\mathrm{w} / \mathrm{v})$ with sterile water. In accordance with the manufacturer's instructions, the corn-soybean meal was mixed with probiotics and incubated at $27-32^{\circ} \mathrm{C}$ for $36 \mathrm{~h}$. We then determined the live bacteria in the fermented product, and the final number of microorganisms was guaranteed at a concentration of $2 \times 10^{9} \mathrm{CFU} / \mathrm{g}$. The method for CFU determination was based on the previous study (Sieuwerts et al., 2008). The 16S rRNA sequences of the bacteria in FF are shown in Supplementary Figure S1. After fermentation, the fermented corn-soybean meal was dried at $30-40^{\circ} \mathrm{C}$ to a moisture content of $10 \%$. The ingredients and nutrient content (\%) of the UF and FF are listed in Table 1. Both the UF and FF met all recommended nutrient levels (NRC, 2012), and neither contained antimicrobials or growth promoters.

\section{Animals, Housing, and Treatment}

A total of 48 growing barrow pigs (Duroc $\times$ Landrace $\times$ Large White) $(53.90 \pm 1.31 \mathrm{~kg}$ initial body weight) were randomly allocated into one of two feeding groups; one group was fed with commercial soybean meal (UF) and a second with fermented complete commercial soybean meal (FF). Each group consisted of 24 barrow pigs that were housed in six pens, with four pigs per pen, in an environmentally controlled facility under a constant temperature of $25-28^{\circ} \mathrm{C}$ and with free access to feed and clean water throughout the experimental period.

TABLE 1 | Ingredients and nutrient components of the experimental meals.

\begin{tabular}{|c|c|c|c|c|c|}
\hline \multirow[t]{2}{*}{ Ingredients } & \multicolumn{2}{|c|}{ Ratio/\% } & \multirow[t]{2}{*}{ Nutrient component $^{2}$} & \multicolumn{2}{|c|}{ Content/\% } \\
\hline & UF & $\mathbf{F F}$ & & UF & FF \\
\hline Corn & 67.0 & 67.0 & Dry matter & 89.42 & 76.03 \\
\hline Soybean meal & 21.0 & 21.0 & Gross energy & 12.78 & 12.65 \\
\hline Wheat bran & 8.0 & 8.0 & Crude protein, & 15.21 & 16.36 \\
\hline Premix ${ }^{1}$ & 4.0 & 4.0 & Crude fiber & 2.48 & 2.96 \\
\hline \multirow[t]{4}{*}{ Total } & 100 & 100 & Crude fat, & 2.02 & 1.40 \\
\hline & & & Crude ash & 5.95 & 5.21 \\
\hline & & & Acid Detergent Fiber & 4.04 & 4.02 \\
\hline & & & Neutral detergent fiber & 13.24 & 11.78 \\
\hline
\end{tabular}

${ }^{1}$ Premix supplied per kilogram of meal: VD 2800 IU; VE, 26 mg; VK, 2 mg; VB1, $50 \mathrm{mg}$; VB6, $3 \mathrm{mg}$; VA, $6480 \mathrm{U}$; VB2, $4 \mathrm{mg}$; VB12, $0.03 \mathrm{mg}$; pantothenic acid, $9 \mathrm{mg}$; nicotinic acid, $20 \mathrm{mg}$; choline chloride, $300 \mathrm{mg}$; biotin, $0.2 \mathrm{mg}$; Fe, $200 \mathrm{mg}$; Cu, 95 mg; Mn, 30 mg; folic acid, 1.2 mg; Zn 100 mg; I, 0.35 mg; Se, 0.36 mg; P $0.1 \% ; \mathrm{NaCl}, 0.5 \%$; lysine, $0.1 \% ; \mathrm{Ca}, 0.9 \% .{ }^{2}$ Nutrient contents are calculated values. 


\section{Sample Collection and Preparation}

Upon reaching slaughter weight (approximately $110 \mathrm{~kg}$ ), the control group was fed UF meal for 76 days and the treatment group was fed FF meal for 56 days. One pig from each pen was randomly selected and fasted for $12 \mathrm{~h}$ (with free access to water) before slaughter and sampling. Blood samples were collected from the external jugular vein into serum separation tubes and centrifuged at $2,500 \mathrm{rpm}$ for $15 \mathrm{~min}$ at $4^{\circ} \mathrm{C}$, then stored at $-80^{\circ} \mathrm{C}$ until analysis. After blood sampling, all 12 pigs were anesthetized and slaughtered. After recovery of the duodenum and colon, duodenal and proximal colonic tissue samples were collected, washed with $0.9 \%$ saline, quickly frozen, and then stored at $-80^{\circ} \mathrm{C}$ until further analysis. Finally, the digesta in the duodenum and colon was immediately collected and frozen at $-80^{\circ} \mathrm{C}$.

\section{Serum Biochemical Indices and Immunoglobulin Analysis}

In the present study, the serum concentrations of aspartate transaminase (AST), alanine transaminase (ALT), total protein, triglycerides, glucose, albumin, globulin, and low-density lipoprotein cholesterol were measured using a Hitachi-7180 Biochemical Analyzer (Hitachi Medical Corporation, Tokyo, Japan) provided by the Yangling Demonstration Zone Hospital. Serum concentrations of immunoglobulin (Ig) A, M, and G (IgA, IgM, and IgG, respectively) were determined with a commercial Enzyme-linked immunosorbent assay kit (BIM Biosciences, San Francisco, CA, United States). All procedures were performed in accordance with the manufacturers' instructions. Each sample was tested in triplicate.

\section{Total RNA Extraction and Real-Time Polymerase Chain Reaction (PCR)}

Total RNA was extracted from liquid nitrogen-frozen samples of the duodenum and colon using TRIzol Regent (Takara Bio Inc., Shiga, Japan). RNA integrity and quality were determined by agarose gel electrophoresis (1\%) and spectrometry (A260/A280), respectively. A commercial reverse transcription (RT) kit (Takara Bio Inc.) was used for the synthesis of cDNA. The RT products (cDNA) were stored at $-20^{\circ} \mathrm{C}$ for relative quantification by PCR. For a real-time quantitative polymerase chain reaction (RTqPCR), every reaction was performed in triplicate using SYBR green kits on an Applied Biosystems ABI 7500 system (Thermo Fisher Scientific, Waltham, MA, United States). The expression levels of all genes were normalized to that of glyceraldehyde 3phosphate dehydrogenase (GAPDH) using the $2^{-\Delta \Delta C T}$ method. The sequences of primers used for RT-qPCR are listed in Table 2.

\section{Intestinal Digesta DNA Extraction and Pyrosequencing}

Total genomic DNA was extracted from the duodenal and colonic digesta samples using the cetyltrimethylammonium bromide and sodium dodecyl sulfate method. DNA concentration and purity were monitored on $1 \%$ agarose gels. DNA extracted from each sample was used as a template to amplify the V4-V5 regions of $16 \mathrm{~S}$ rRNA genes for later pyrosequencing by Novogene
TABLE 2 | Primers sequences for RT-qPCR.

\begin{tabular}{|c|c|c|c|}
\hline Genes & Primer sequence $\left(5^{\prime}-3^{\prime}\right)$ & $\begin{array}{l}\text { Product } \\
\text { (bp) }\end{array}$ & $\begin{array}{l}\text { GenBank } \\
\text { accession }\end{array}$ \\
\hline \multirow[t]{2}{*}{ TLR1 } & F:TTAGGAGACTCTTACGGGGAA & 135 & NM_001031775.1 \\
\hline & R:ATTTACTGCGGTGCTGACTGA & & \\
\hline \multirow[t]{2}{*}{ TLR2 } & F:GTITACGGAAATTGTGAAACTG & 128 & NM_213761.1 \\
\hline & R:TCCACATTACCGAGGGATTT & & \\
\hline \multirow[t]{2}{*}{ TLR3 } & F:GCATTGCCTGGTTTGTTAGTTG & 122 & NM_001097444.1 \\
\hline & R:TGTATCAAAAAGAATCACTGGGAG & & \\
\hline \multirow[t]{2}{*}{ TLR4 } & F:ATATGGCAGAGGTGAAAGCAC & 125 & NM_001113039.2 \\
\hline & R:GAAGGCAGAGATGAAAAGGGG & & \\
\hline \multirow[t]{2}{*}{ TLR5 } & F:AGTTCCGGGGATTTGTTCA & 110 & NM_001348771.1 \\
\hline & R:GCATAAGTAGGCATCGTATTTGTAT & & \\
\hline \multirow[t]{2}{*}{ TLR6 } & F:CATCACCAGCCTCAAGCATTT & 90 & NM_213760.2 \\
\hline & R:TTCAGTTGTGTCAAGTTGCCAA & & \\
\hline \multirow[t]{2}{*}{ TLR7 } & F:ATAGCGAGCATCACTCCAGCC & 127 & NM_001097434.1 \\
\hline & R:TAATCTGCTGCCTTCTGGTGC & & \\
\hline \multirow[t]{2}{*}{ TLR8 } & F:CTGGGATGCTTGGTTCATCT & 150 & NM_214187.1 \\
\hline & R:CATGAGGTTGTCGATGATGG & & \\
\hline \multirow[t]{2}{*}{ TLR9 } & F:ACAATGACATCCATAGCCGAGT & 80 & NM_213958.1 \\
\hline & R:CAGATCGTTGCCGCTAAAGT & & \\
\hline \multirow[t]{2}{*}{$P B D-1$} & F:TGCCACAGGTGCCGATCT & 105 & NM_213838.1 \\
\hline & R:CTGTTAGCTGCTTAAGGAATAAAGGC & & \\
\hline \multirow[t]{2}{*}{ PR39 } & F:CCACTCCATCACCGTITICC & 129 & NM_214450.1 \\
\hline & R:CAAGGCCACCTCCGTIT & & \\
\hline \multirow[t]{2}{*}{ GAPDH } & F:AGGTCGGAGTGAACGGATTTG & 118 & NM_001206359.1 \\
\hline & R:ACCATGTAGTGGAGGTCAATGAAG & & \\
\hline
\end{tabular}

F, forward; $R$, reverse; $P B D-1$, porcine beta-defensin 1; PR39, proline-arginine rich 39-amino acid peptide; TLR, Toll-like receptor; GAPDH, glyceraldehyde-3phosphate dehydrogenase.

Biological Information Technology Co. (Beijing, China), as described previously (Tian and Zhang, 2017). The forward and reverse primer sequences for the V4-V5 rRNA gene library preparation are presented in Supplementary Table S1. Raw reads were submitted to the Sequence Read Archive of the National Center for Biotechnology Information (SRA, No. PRJNA524989).

\section{Bioinformatics Analyses}

The paired-end reads were merged using FLASH (V1.2.7) (Magoc and Salzberg, 2011). Quality filtering on the raw tags was performed according to the QIIME 1.7.0 ${ }^{2}$ quality controlled process (Caporaso et al., 2010). Next, the tags were compared with the reference database (Gold database) ${ }^{3}$ (Edgar et al., 2011) using an UCHIME algorithm (UCHIME Algorithm) ${ }^{4}$, to detect chimera sequences, and the chimera sequences were then removed (Haas et al., 2011). The Effective Tags were then finally obtained. High-quality clean tags were obtained and classified into the same operational taxonomic units (OTUs) at an identity threshold of $97 \%$ similarity by UPARSE software (Uparse

\footnotetext{
${ }^{1}$ http://ccb.jhu.edu/software/FLASH/

${ }^{2} \mathrm{http}: / /$ qiime.org/index.html

${ }^{3} \mathrm{http}: / /$ drive5.com/uchime/uchime_download.html

${ }^{4} \mathrm{http}: / /$ www.drive5.com/usearch/manual/uchime_algo.html
} 
v7.0.1001 $)^{5}$ (Edgar, 2013). For each representative sequence, the Greengene Database ${ }^{6}$ (DeSantis et al., 2006) was used based on a ribosomal database projects (RDPs) classifier (Version 2.2) (Wang et al., 2007) algorithm to annotate taxonomic information. In order to study the phylogenetic relationship of different OTUs, and the difference of the dominant species in different samples (groups), multiple sequence alignments were conducted using MUSCLE software (Version 3.8.31) ${ }^{8}$ (Edgar, 2004). OTUs abundance information was normalized using a standard of sequence number corresponding to the sample with the fewest number of sequences. Subsequent analyses of alpha diversity and beta diversity were all performed based on this normalized output data. Alpha diversity is applied in analyzing the complexity of species diversity for a sample through four indices, including Observed species, Chao 1, Shannon, Simpson, and ACE. All the indices in our samples were calculated with QIIME (Version 1.7.0) and displayed with $\mathrm{R}$ software (Version 2.15.3). The relative abundance at the phylum and genus levels was compared between the two groups, the top 30 most abundant families were defined as predominant genera and sorted for the comparison.

\section{Untargeted Metabolomics Study Based on Liquid Chromatography Tandem Mass Spectrometry (LC-MS/MS)}

Tissues (100 mg) were individually ground with liquid nitrogen and the homogenate was resuspended in pre-chilled $80 \%$ methanol $\left(-20^{\circ} \mathrm{C}\right)$ and then vortexed. The samples were incubated at $-20^{\circ} \mathrm{C}$ for $60 \mathrm{~min}$ and then centrifuged at $14,000 \mathrm{~g}$ and $4^{\circ} \mathrm{C}$ for $20 \mathrm{~min}$. The supernatants were subsequently transferred to a fresh Eppendorf tube and spun in a vacuum concentrator until dry. The dried metabolite pellets were reconstituted in $60 \%$ methanol and analyzed by LC-MS/MS.

LC-MS/MS analyses were performed using a Vanquish ultrahigh-performance liquid chromatography (UHPLC) system (Thermo Fisher Scientific) coupled with an Orbitrap Q Exactive HF-X mass spectrometer (Thermo Fisher Scientific) at Novogene Genetics, Beijing, China. Samples were injected into a Hypersil Gold column $(100 \times 2.1 \mathrm{~mm}, 1.9 \mu \mathrm{m}$; Thermo Fisher Scientific $)$ using a $16 \mathrm{~min}$ linear gradient at a flow rate of $0.3 \mathrm{~mL} / \mathrm{min}$. The eluents for the positive polarity mode were eluent A $(0.1 \%$ aqueous formic acid solution) and eluent B (methanol). The eluents for the negative polarity mode were eluent A $(5 \mathrm{mmol} / \mathrm{L}$ ammonium acetate, $\mathrm{pH} 9.0$ ) and eluent $\mathrm{B}$ (methanol). The solvent gradient was set as follows: $2 \% \mathrm{~B}, 1.5 \mathrm{~min} ; 2-100 \% \mathrm{~B}, 12.0 \mathrm{~min}$; $100 \% \mathrm{~B}, 14.0 \mathrm{~min} ; 100-2 \% \mathrm{~B}, 14.1 \mathrm{~min}$; and $2 \% \mathrm{~B}, 16.0 \mathrm{~min} . \mathrm{A} \mathrm{Q}$ Exactive HF-X mass spectrometer (Thermo Fisher Scientific) was operated in positive/negative polarity mode with a spray voltage of $3.2 \mathrm{kV}$, capillary temperature of $320^{\circ} \mathrm{C}$, sheath gas flow rate of $35 \mathrm{arb}$, and auxiliary gas flow rate of $10 \mathrm{arb}$.

The raw data files generated by UHPLC-MS/MS were processed using the Compound Discoverer 3.0 (Thermo Fisher

\footnotetext{
${ }^{5}$ http://drive5.com/uparse/

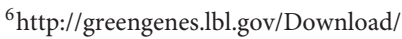

${ }^{7}$ http://sourceforge.net/projects/rdp-classifier/

${ }^{8}$ http://www.drive5.com/muscle/
}

Scientific) to perform peak alignment, peak picking, and quantitation of each metabolite. The main parameters were set as follows: retention time tolerance, $0.2 \mathrm{~min}$; actual mass tolerance, $5 \mathrm{ppm}$; signal intensity tolerance, 30\%; signal/noise ratio, 3; and minimum intensity, 100000. Afterward, peak intensities were normalized to the total spectral intensity. The normalized data were used to predict the molecular formula based on additive ions, molecular ion peaks, and fragment ions. Then, the peaks were matched with the mzCloud $^{9}$ and ChemSpider ${ }^{10}$ databases to obtain accurate qualitative and relative quantitative results. The online KEGG database was used to identify metabolites by matching the molecular mass data. Finally, metabolites for separating the models were selected with the following requirements: variable importance in projection (VIP) $>1$ and $\mid \mathrm{P}($ corr $) \mid \geq 0.5$ with $95 \%$ jack-knifed confidence intervals. The Student's $t$-test was applied to further analyze intergroup significance of the selected metabolites. Pathway analysis and enrichment analysis of differential metabolites were conducted on the MetaboAnalyst web server ${ }^{11}$.

\section{Statistical Analysis}

Data are presented as the mean \pm standard error of the mean (SEM). All statistical analyses were performed using IBM SPSS Statistics for Windows, version 19.0 (IBM Corporation, Armonk, NY, United States) using an one-way ANOVA with Turkey's multiple comparison test or Student's test. A probability $(P)$ value of $<0.05$ was considered statistically significant. The correlations between the colonic microbial composition (relative abundance of family higher than $0.1 \%$ ) and serum immunity and genes related to gut immunity that were significantly affected by FF treatment were assessed by a Spearman's correlation test using GraphPad Prism version 8.00 (GraphPad Software, San Diego, CA, United States). The correlations between the colonic microbial composition (relative abundance of family higher than $0.1 \%$ ) and metabolites that were significantly affected by FF meal were assessed by a Spearman's correlation analysis using GraphPad Prism version 8.00 (GraphPad Software, San Diego, CA, United States).

\section{RESULTS}

\section{FF Increased Growth Performance}

To investigate the effect of FF on body weight, after reaching the similar weight $(53.19 \pm 2.17 \mathrm{~kg}$ vs. $54.60 \pm 1.62 \mathrm{~kg})$, the pigs fed a corn-soybean meal were given free access to FF or UF. When compared with the UF group, the average daily weight gain was significantly increased in the FF group $(P<0.05)$ and there was no significant difference $(P>0.05)$ in the average daily intake among the two groups. Furthermore, the weight gain to food ratio was significantly increased in the FF group rather than in the UF group $(P<0.05)$ (Table 3$)$.

\footnotetext{
${ }^{9}$ https://www.mzcloud.org/

${ }^{10} \mathrm{http}: / /$ www.chemspider.com/

${ }^{11}$ https://www.metaboanalyst.ca/
} 
TABLE 3 | Effects of fermented meal on growth performance of grower-finisher pigs.

\begin{tabular}{lrrr}
\hline Items & \multicolumn{1}{c}{ UF } & \multicolumn{1}{c}{ FF } & P-value \\
\hline Initial body weight $(\mathrm{kg})$ & $53.19 \pm 2.17$ & $54.60 \pm 1.62$ & 0.615 \\
Final body weight $(\mathrm{kg})$ & $115.34 \pm 2.33$ & $116.76 \pm 2.04$ & 0.606 \\
Average daily gain $\left(\mathrm{kg} \cdot \mathrm{d}^{-1}\right)$ & $0.74 \pm 0.06$ & $1.04 \pm 0.04$ & 0.013 \\
Average daily intake $\left(\mathrm{kg} \cdot \mathrm{d}^{-1}\right)$ & $2.20 \pm 0.07$ & $2.31 \pm 0.07$ & 0.350 \\
Gain:food ratio & $0.33 \pm 0.02$ & $0.45 \pm 0.03$ & 0.021 \\
\hline
\end{tabular}

Data are presented as the mean \pm SEM $(n=6)$. UF, pigs fed with unfermented corn-soybean meal; FF, pigs fed with fermented corn-soybean meal.

\section{FF Changed Serum Immunity}

Next, the effects of FF on immunity were assessed. As shown in Figures $\mathbf{1 A}-\mathrm{C}$, the ALT and AST concentrations tended to decrease in the FF-treated group as compared to the UF group $(P>0.05)$. There were no significant differences in the other serum parameters between the two groups (Supplementary Table S2). As shown in Figures 1D-F, FF significantly increased the serum concentrations of $\operatorname{IgM}$ and $\operatorname{IgG}(P<0.05)$, while there was no significant difference in IgA levels between the two meals.

\section{Effect of FF on the Expression of Genes Related to Gut Immunity}

The effects of FF on the expression of genes related to gut immunity was determined. The expression levels of the antimicrobial peptide-encoding genes $P B D-1$ and $P R 39$ revealed the potential capacity for the eradication of invading pathogens. The results of the gene expression analysis of the intestinal tissue are presented in Figure 2. The FF meal significantly increased the mRNA abundance of AMPs and TLRs. In the duodenum, the mRNA abundances of TLR1, TLR2, TLR3, TLR4, $P B D-1$, and $P R-39$ in the FF-treated pigs were significantly increased $(P<0.05)$, while those of TLR5 and TLR9 were very significantly increased $(P<0.01$; Figure $2 \mathrm{~A})$. In the proximal colon, the mRNA abundances of TLR4 and TLR5 in the FF-treated pigs were significantly increased $(P<0.05)$, while those of TLR1, TLR2, TLR7, and PBD-1 in the FFtreated pigs were very significantly increased $(P<0.01$; Figure 2B).

\section{FF Shaped the Intestinal Microbiota}

To explore the cause of improved immunity in FF-treated pigs, the intestinal microbiota were quantified by $16 \mathrm{~S}$ rRNA sequence analysis, which identified a total of $65,249 \pm 16,236 \mathrm{~V} 4-\mathrm{V} 5$ $16 \mathrm{~S}$ rRNA sequence reads from each sample. Binning sequences using a pairwise identity threshold of $97 \%$ obtained an average of $800 \pm 231$ operational taxonomic units per sample. MOTHUR plotting for the construction of a sparse curve between the number of reads and the number of operational taxonomic units revealed a tendency for plateau saturation (Supplementary Figure S2). Statistical estimates of species richness for 5,000 subsets of each sample at a genetic distance of 3\% showed
A

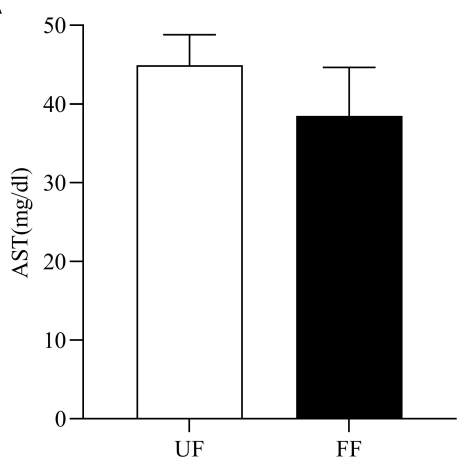

D

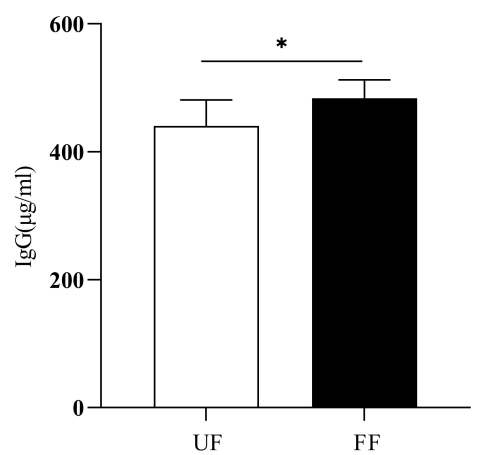

B

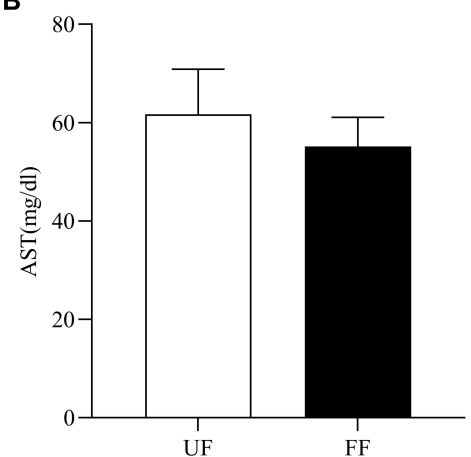

E

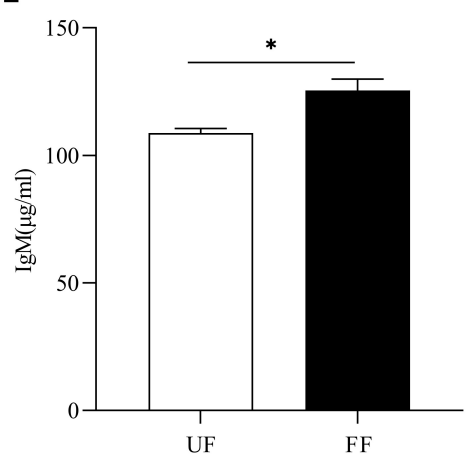

C

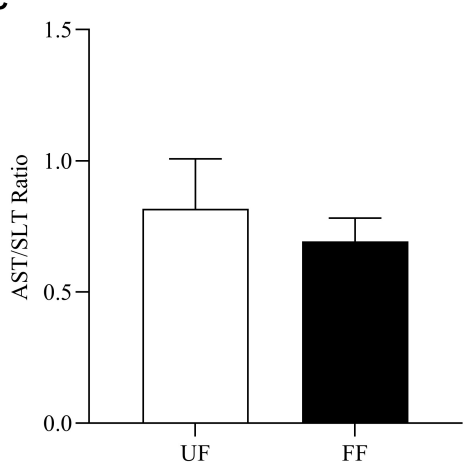

$\mathbf{F}$

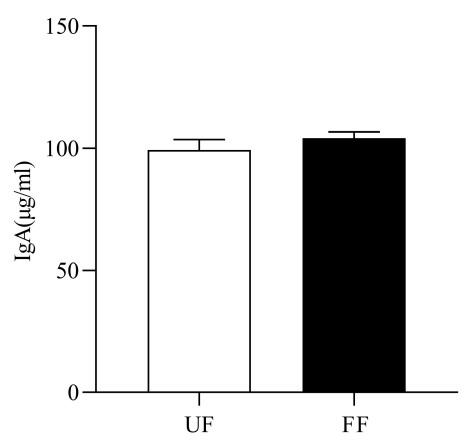

FIGURE 1 | FF changed serum immunity. (A) AST, (B) ALT, (C) AST/ALT ratio, (D-F). Serum immunoglobulins, IgM (D), IgA (E), and IgG (F). UF, pigs fed with unfermented corn-soybean meal; FF, pigs fed with fermented corn-soybean meal. ${ }^{*} P<0.05$. 


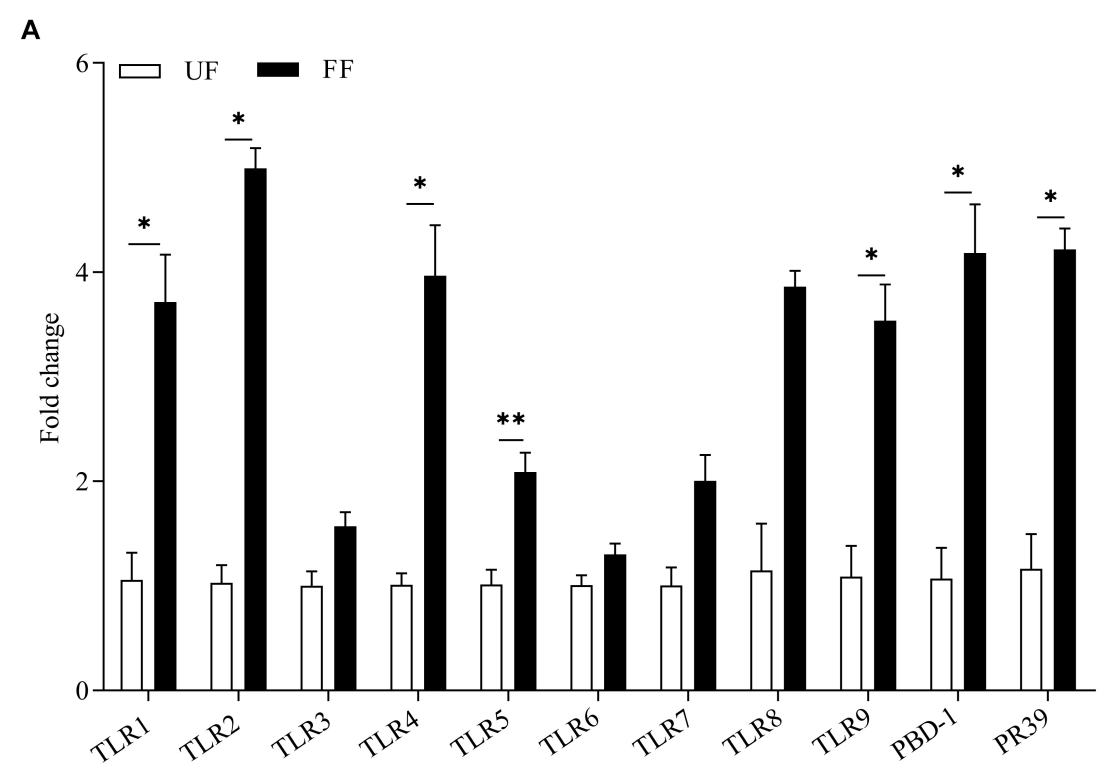

B

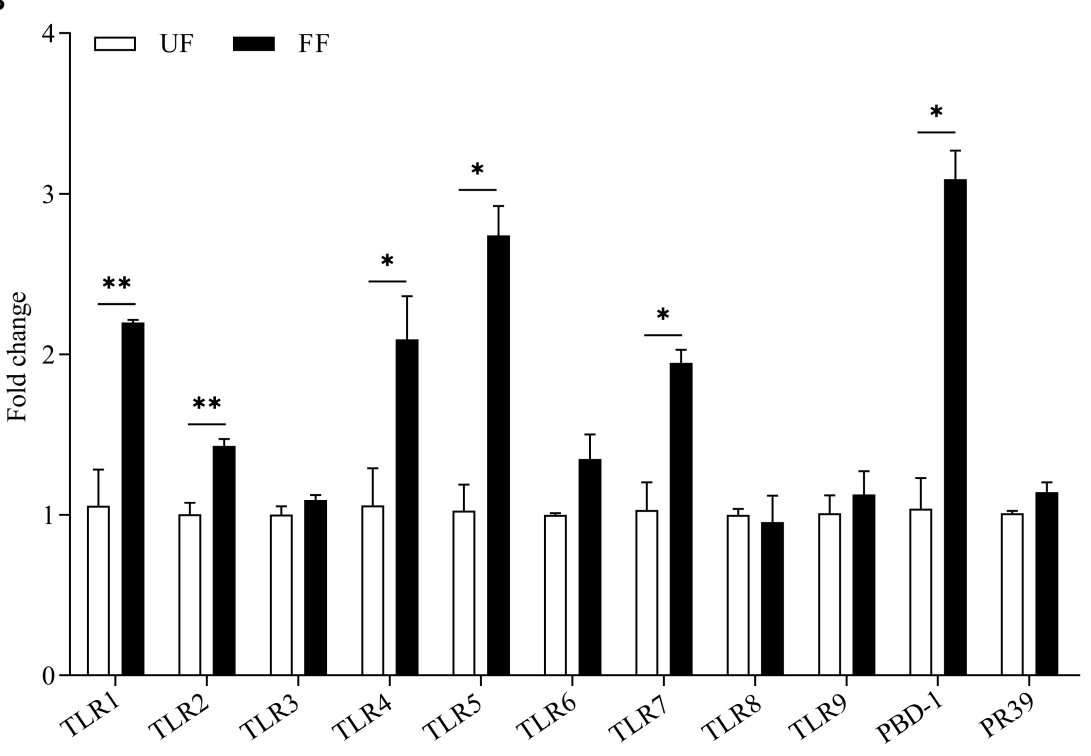

FIGURE 2 | Expression levels of TLR genes and antimicrobial peptides in the duodenum (A) and colon (B). The UF group was designed as one-fold change. UF, pigs fed with unfermented corn-soybean meal; FF, pigs fed with fermented corn-soybean meal. Data are presented as the mean $\pm \mathrm{SEM}(n=6)$. ${ }^{*}<0.05$ and $* * P<0.01$.

that there was no difference in richness estimators (ACE and Chao 1) and the diversity indices (Shannon and Simpson diversity) (Table 4).

At the phylum level, Firmicutes and Bacteroidetes were the most predominant phyla in the duodenum and colon (Tables 5, 6). FF meal significantly decreased the proportion of Bacteroidetes and Verrucomicrobia $(P<0.05)$ and tended to decrease the relative abundance of Spirochaetes in the duodenum $(P=0.069)$ (Table 5). FF meal significantly increased the proportion of Firmicutes and Actinobacteria $(P<0.01)$, whereas the proportion of Proteobacteria, Bacteroidetes, and Verrucomicrobia was decreased in the colon $(P<0.05)$ (Table 6).
A family-level analysis of the top 30 most abundant families revealed that Acidaminococcaceae and Clostridiales_vadinBB60_group were significantly decreased in relative abundance by the FF meal treatment in the duodenum $(P<0.05)$ (Table 7). A family-level analysis of the top 30 most abundant families revealed that Ruminococcaceae, Rikenellaceae, Christensenellaceae, Lactobacillaceae, and Family XIII were significantly increased in relative abundance by the FF meal treatment, whereas the abundance of Prevotellaceae, Lachnospiraceae, Clostridiaceae_1, Bacteroidales_RF16_group, Streptococcaceae, Veillonellaceae, Erysipelotrichaceae, Peptostreptococcaceae, Acidaminococcaceae, Bacteroidaceae, 
TABLE 4 | Diversity estimation of the 16S rRNA gene libraries from microbiota in the duodenum and colon of pigs fed the control UF and FF meals.

\begin{tabular}{|c|c|c|c|c|c|c|}
\hline \multirow[t]{2}{*}{ Items } & \multicolumn{3}{|c|}{ Duodenum } & \multicolumn{3}{|c|}{ Colon } \\
\hline & UF & FF & $P$-value & UF & $\mathbf{F F}$ & $P$-value \\
\hline Ace & $734.452 \pm 255.71$ & 563.738 土 156.54 & 0.142 & $1116.32 \pm 84.48$ & $968.950 \pm 45.31$ & 0.132 \\
\hline Chao 1 & $719.902 \pm 237.37$ & $542.699 \pm 149.85$ & 0.148 & $1120.88 \pm 83.80$ & $970.55 \pm 42.16$ & 0.201 \\
\hline Shannon & $3.573 \pm 0.86$ & $3.132 \pm 1.79$ & 0.191 & $7.63 \pm 0.31$ & $7.12 \pm 0.86$ & 0.918 \\
\hline Simpson & $0.721 \pm 0.08$ & $0.637 \pm 0.31$ & 0.332 & $0.99 \pm 0.04$ & $0.96 \pm 0.07$ & 0.993 \\
\hline
\end{tabular}

UF, pigs fed with unfermented corn-soybean meal; FF, pigs fed with fermented corn-soybean meal. Data are presented as the mean \pm SEM ( $n=6)$.

Succinivibrionaceae,

Anaeroplasmataceae, Rhodospirillaceae, and Unidentified_Thermoplasmatales was decreased in the colon $(P<0.05)$ (Table 8).

\section{Correlation Between Colonic Microbial Composition and Serum Immunity and Genes Related to Gut Immunity}

To comprehensively analyze the relations between host serum immunity, genes related to gut immunity, and gut microbiota, a correlation matrix was generated by

TABLE 5 | Relative abundance of microbial phylum (percentage) in the duodenum of pigs in the FF and control groups.

\begin{tabular}{lccc}
\hline Items & UF & FF & $\boldsymbol{P}$-value \\
\hline Firmicutes & $51.29 \pm 11.11$ & $78.61 \pm 14.67$ & 0.168 \\
Proteobacteria & $1.15 \pm 0.54$ & $17.28 \pm 15.35$ & 0.341 \\
Bacteroidetes & $33.26 \pm 11.16$ & $1.10 \pm 0.37$ & 0.035 \\
Actinobacteria & $0.95 \pm 0.81$ & $2.06 \pm 0.84$ & 0.363 \\
Unclassified phylum & $11.18 \pm 7.16$ & $0.02 \pm 0.01$ & 0.180 \\
Tenericutes & $1.22 \pm 0.63$ & $0.47 \pm 0.04$ & 0.348 \\
Planctomycetes & $0.01 \pm 0.01$ & $0.05 \pm 0.03$ & 0.366 \\
Verrucomicrobia & $0.07 \pm 0.02$ & $0.01 \pm 0.00$ & 0.037 \\
Euryarchaeota & $0.01 \pm 0.00$ & $0.01 \pm 0.00$ & 0.397 \\
Spirochaetes & $0.64 \pm 0.26$ & $0.03 \pm 0.01$ & 0.069
\end{tabular}

UF, pigs fed with unfermented corn-soybean meal; FF, pigs fed with fermented corn-soybean meal. Data are presented as the mean \pm SEM $(n=6)$.

TABLE 6 | Relative abundance of microbial phylum (percentage) in the colon of pigs in the FF and control groups.

\begin{tabular}{lrrr}
\hline Items & \multicolumn{1}{c}{ UF } & \multicolumn{1}{c}{ FF } & $\boldsymbol{P}$-value \\
\hline Firmicutes & $40.42 \pm 1.94$ & $62.64 \pm 3.52$ & 0.001 \\
Proteobacteria & $1.68 \pm 0.13$ & $0.20 \pm 0.05$ & $<0.001$ \\
Bacteroidetes & $51.59 \pm 2.29$ & $31.78 \pm 3.27$ & 0.001 \\
Actinobacteria & $0.15 \pm 0.02$ & $0.50 \pm 0.10$ & 0.020 \\
Unclassified phylum & $0.08 \pm 0.02$ & $0.04 \pm 0.01$ & 0.085 \\
Tenericutes & $2.27 \pm 0.33$ & $1.59 \pm 0.23$ & 0.129 \\
Planctomycetes & $0.02 \pm 0.01$ & $0.49 \pm 0.23$ & 0.095 \\
Verrucomicrobia & $0.57 \pm 0.12$ & $0.01 \pm 0.00$ & 0.005 \\
Euryarchaeota & $0.11 \pm 0.03$ & $0.18 \pm 0.15$ & 0.689 \\
Spirochaetes & $2.59 \pm 0.30$ & $2.50 \pm 0.63$ & 0.910
\end{tabular}

UF, pigs fed with normal commercial feed; FF, pigs fed with fermented meal. Data are presented as the mean $\pm \operatorname{SEM}(n=6)$. calculating the Spearman's correlation coefficient. As shown in Figure 3, Spearman's correlation analysis showed that the serum IgM was positively associated with the abundance of Enterobacteriaceae but negatively related to the proportion of Lachnospiraceae in the colon. The gene expression of TLR1 was negatively correlated with the abundance of Ruminococcaceae and Erysipelotrichaceae. The gene expression of TLR2 was positively associated with the abundance of Veillonellaceae. The gene expression of TLR3 was negatively correlated with the abundance of Peptostreptococcaceae. The gene expression of TLR4 was positively correlated with the abundance of Enterobacteriaceae, Clostridiaceae_1, and Bacteroidales_S24-7_group. The gene expression of TLR5 and PBD-1 were positively associated with the abundance Lachnospiraceae but negatively related to the proportion of Bacteroidales_S24-7_group. The gene expression of TLR7 was positively associated with the abundance Bacteroidales_S24-7_group but negatively related to the proportion of Lachnospiraceae. Additionally, the gene expression of TLR5 was also negatively related to the proportion of Veilonellaceae.

\section{Effect of FF on Fermentation Metabolites in Colonic Digesta}

To further study the effect of FF on the gut, the colonic digesta metabolic profiles of the two meals were acquired by LC-MS. As shown by the PCA score plots presented in Figures $\mathbf{4 A , B}$ which distinguished metabolic communities based on meal of colonic sampling, the metabolic communities were clustered. The partial least squares discriminant analysis (PLS-DA) score plots also reflected that $\mathrm{FF}$ led to significant biochemical changes (Figures 4C,D). Furthermore, LC-MS/MS (ESI-) and LCMS/MS (ESI+) detected a total of 398 and 953 biomarker metabolites, respectively (Figures $4 \mathrm{E}, \mathrm{F}$ ).

In summary, there were significant differences in the 1,351 biomarker metabolites between the UF and FF groups (detailed information is presented in Supplementary Data S1). Next, the Kyoto Encyclopedia of Genes and Genomes (KEGG) was used to analyze the pathways of the metabolites that differed between the two meal groups (Figure 5). The effects of C5-branched dibasic acid metabolism were significantly upregulated while the purine metabolism was significantly downregulated in the $\mathrm{FF}$ group, as compared to the control group $(P<0.05)$. Meanwhile, the effects of dioxin degradation, phenylpropanoid biosynthesis, and aminobenzoate degradation were downregulated in the FF group $(0.05<P<0.1)$. 
TABLE 7 | Relative abundance (percentage) for the top 30 most abundant family in the duodenum of pigs in the fermented feed (FF) and control (UF) groups.

\begin{tabular}{|c|c|c|c|}
\hline Items & UF & FF & $P$-value \\
\hline Prevotellaceae & $23.85 \pm 10.94$ & $0.33 \pm 0.13$ & 0.084 \\
\hline Clostridiaceae_1 & $13.72 \pm 9.68$ & $7.26 \pm 5.81$ & 0.582 \\
\hline Unclassified family & $12.74 \pm 6.79$ & $0.79 \pm 0.46$ & 0.139 \\
\hline Lactobacillaceae & $11.52 \pm 10.01$ & $42.17 \pm 15.73$ & 0.137 \\
\hline Ruminococcaceae & $10.63 \pm 6.04$ & $4.75 \pm 4.22$ & 0.446 \\
\hline Veillonellaceae & $5.54 \pm 2.40$ & $8.78 \pm 3.60$ & 0.474 \\
\hline Lachnospiraceae & $4.55 \pm 1.50$ & $1.84 \pm 1.61$ & 0.246 \\
\hline Bacteroidales_S24-7_group & $3.87 \pm 2.15$ & $0.22 \pm 0.14$ & 0.150 \\
\hline Rikenellaceae & $2.16 \pm 0.97$ & $0.10 \pm 0.03$ & 0.087 \\
\hline Porphyromonadaceae & $1.34 \pm 0.66$ & $0.11 \pm 0.04$ & 0.119 \\
\hline Peptostreptococcaceae & $1.28 \pm 0.38$ & $4.57 \pm 3.28$ & 0.362 \\
\hline Bacteroidales_RF16_group & $1.21 \pm 0.64$ & $0.04 \pm 0.02$ & 0.125 \\
\hline Erysipelotrichaceae & $0.99 \pm 0.27$ & $3.67 \pm 2.48$ & 0.329 \\
\hline Nocardiaceae & $0.78 \pm 0.78$ & $0.03 \pm 0.02$ & 0.381 \\
\hline Streptococcaceae & $0.74 \pm 0.30$ & $0.18 \pm 0.08$ & 0.122 \\
\hline Acidaminococcaceae & $0.74 \pm 0.16$ & $0.06 \pm 0.02$ & 0.009 \\
\hline Spirochaetaceae & $0.64 \pm 0.26$ & $0.03 \pm 0.01$ & 0.069 \\
\hline Christensenellaceae & $0.64 \pm 0.33$ & $1.08 \pm 0.96$ & 0.681 \\
\hline Family XIII & $0.52 \pm 0.34$ & $0.40 \pm 0.36$ & 0.823 \\
\hline Bacteroidaceae & $0.40 \pm 0.21$ & $0.04 \pm 0.01$ & 0.147 \\
\hline Campylobacteraceae & $0.20 \pm 0.10$ & $0.01 \pm 0.01$ & 0.131 \\
\hline Mycoplasmataceae & $0.19 \pm 0.17$ & $0.00 \pm 0.00$ & 0.296 \\
\hline Neisseriaceae & $0.18 \pm 0.12$ & $0.07 \pm 0.04$ & 0.414 \\
\hline Alcaligenaceae & $0.17 \pm 0.11$ & $0.02 \pm 0.01$ & 0.240 \\
\hline Clostridiales_vadinBB60_group & $0.13 \pm 0.05$ & $0.00 \pm 0.00$ & 0.040 \\
\hline Burkholderiaceae & $0.12 \pm 0.12$ & $0.01 \pm 0.00$ & 0.370 \\
\hline Anaeroplasmataceae & $0.11 \pm 0.05$ & $0.00 \pm 0.00$ & 0.063 \\
\hline Peptococcaceae & $0.11 \pm 0.07$ & $0.02 \pm 0.02$ & 0.269 \\
\hline Helicobacteraceae & $0.10 \pm 0.05$ & $0.00 \pm 0.00$ & 0.147 \\
\hline P-2534-18B5_gut_group & $0.09 \pm 0.03$ & $0.01 \pm 0.00$ & 0.060 \\
\hline
\end{tabular}

UF, pigs fed with unfermented corn-soybean meal; FF, pigs fed with fermented corn-soybean meal. Data are presented as the mean $\pm \operatorname{SEM}(n=6)$.

\section{Correlation Between Microbiota Communities and Related Metabolites}

Metabolomics has been shown to be an important tool to reveal the potential crosstalk of host and gut microbiota. Therefore, correlations between metabolites and familylevel microbiota with significant differences between two meals were obtained via spearman's correlation analysis (Figure 6). As shown in Figure 6, ten bacterial strains (family Enterobacteriaceae, Lactobacillaceae, Prevotellaceae, Clostridiaceae_1, Ruminococcaceae, Veillonellaceae, Peptostreptococcaceae, Erysipelotrichaceae, Lachnospiraceae, and Bacteroidales_S24-7_group) were most closely related to the metabolites in the FF group, as compared to the control group $(P<0.05)$.

\section{DISCUSSION}

The gut microbiota are critical to metabolism, nutrient absorption, and host immunity (El Aidy et al., 2013), and the pig
TABLE 8 | Relative abundance (percentage) for the top 30 most abundant family in the colon of pigs in the fermented feed (FF) and control (UF) groups.

\begin{tabular}{|c|c|c|c|}
\hline Items & UF & FF & $P$-value \\
\hline Prevotellaceae & $28.31 \pm 4.11$ & $9.54 \pm 1.13$ & 0.005 \\
\hline Ruminococcaceae & $13.22 \pm 1.18$ & $31.78 \pm 3.48$ & 0.002 \\
\hline Lachnospiraceae & $11.77 \pm 0.82$ & $8.41 \pm 0.84$ & 0.017 \\
\hline Bacteroidales_S24-7_group & $11.29 \pm 1.47$ & $8.18 \pm 0.73$ & 0.099 \\
\hline Rikenellaceae & $4.63 \pm 0.25$ & $8.89 \pm 1.27$ & 0.020 \\
\hline Unclassified family & $3.97 \pm 0.42$ & $2.52 \pm 0.31$ & 0.022 \\
\hline Clostridiaceae_1 & $3.43 \pm 0.49$ & $0.69 \pm 0.14$ & 0.002 \\
\hline Bacteroidales_RF16_group & $3.12 \pm 0.36$ & $1.50 \pm 0.29$ & 0.006 \\
\hline Streptococcaceae & $2.92 \pm 0.92$ & $0.01 \pm 0.01$ & 0.025 \\
\hline Spirochaetaceae & $2.59 \pm 0.30$ & $2.50 \pm 0.63$ & 0.910 \\
\hline Veillonellaceae & $2.42 \pm 0.39$ & $0.16 \pm 0.05$ & 0.002 \\
\hline Porphyromonadaceae & $2.09 \pm 0.30$ & $1.84 \pm 0.40$ & 0.628 \\
\hline Erysipelotrichaceae & $1.45 \pm 0.07$ & $0.87 \pm 0.12$ & 0.002 \\
\hline Peptostreptococcaceae & $1.32 \pm 0.13$ & $0.91 \pm 0.12$ & 0.040 \\
\hline Acidaminococcaceae & $1.20 \pm 0.11$ & $0.57 \pm 0.12$ & 0.004 \\
\hline Bacteroidaceae & $0.95 \pm 0.15$ & $0.38 \pm 1.44$ & 0.012 \\
\hline Christensenellaceae & $0.86 \pm 0.17$ & $7.36 \pm 6.65$ & 0.006 \\
\hline Lactobacillaceae & $0.66 \pm 0.17$ & $9.95 \pm 1.44$ & 0.221 \\
\hline Family XIII & $0.45 \pm 0.04$ & $1.16 \pm 0.16$ & 0.006 \\
\hline Succinivibrionaceae & $0.40 \pm 0.10$ & $0.00 \pm 0.00$ & 0.010 \\
\hline Clostridiales_vadinBB60_group & $0.40 \pm 0.04$ & $0.04 \pm 0.01$ & $<0.001$ \\
\hline Anaeroplasmataceae & $0.39 \pm 0.04$ & $0.02 \pm 0.01$ & $<0.001$ \\
\hline Bacteroidales_BS11_gut_group & $0.30 \pm 0.12$ & $0.58 \pm 0.33$ & 0.450 \\
\hline Alcaligenaceae & $0.27 \pm 0.03$ & $0.01 \pm 0.00$ & $<0.001$ \\
\hline Campylobacteraceae & $0.21 \pm 0.09$ & $0.01 \pm 0.00$ & 0.072 \\
\hline P-2534-18B5_gut_group & $0.20 \pm 0.04$ & $0.31 \pm 0.03$ & 0.065 \\
\hline Fibrobacteraceae & $0.14 \pm 0.03$ & $0.03 \pm 0.01$ & 0.019 \\
\hline Rhodospirillaceae & $0.14 \pm 0.03$ & $0.01 \pm 0.00$ & 0.003 \\
\hline Peptococcaceae & $0.11 \pm 0.02$ & $0.15 \pm 0.02$ & 0.186 \\
\hline Unidentified_Thermoplasmatales & $0.10 \pm 0.03$ & $0.00 \pm 0.00$ & 0.017 \\
\hline
\end{tabular}

UF, pigs fed with normal commercial feed; FF, pigs fed with fermented meal. Data are presented as the mean \pm SEM $(n=6)$.

microbiota has become the focus of much attention (Frese et al., 2015; Ji et al., 2018; Wang W. et al., 2019). FF, as an available feed alternative, has great potential to improve gut health and maintain gastrointestinal tract microbial homeostasis (Jin et al., 2017) and could modulate the host gut microbiota through dietary manipulation (Wang et al., 2018). In this study, the effects of FF versus normal feed on serum immunity, expression of genes related to gut immunity, gut microbiota composition, and bacterial metabolites were investigated in grower-finisher pigs. The results reflected that FF regulated the microbiota composition in the duodenum and colon of pigs, and it also selectively changed the metabolomics profiles.

Corn-soybean meal is the most frequently used meal for livestock production in China. Solid-state fermentation can improve the nutritional value of plant materials and has it been suggested to increase the use of FF in livestock feeds (Shi et al., 2017). In the animal production, pigs are often slaughtered at a constant body weight (market weight, around $110 \mathrm{~kg}$ ) to maintain uniformity of pork products and maximize profits (Kim et al., 2005; Frederick et al., 2006; Vermeer et al., 2014). In the present 


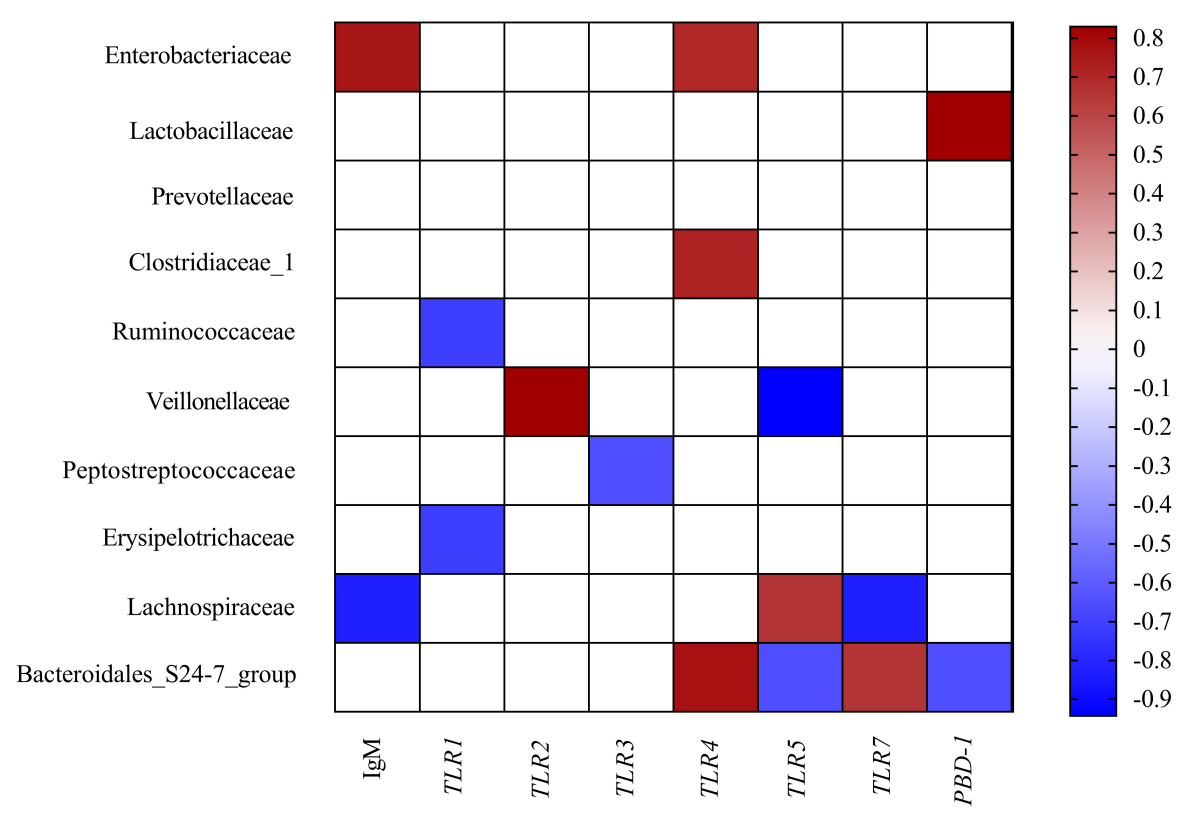

FIGURE 3 | Correlation analysis between colonic microbial composition (relative abundance of family higher than $0.1 \%$ ) and serum immunity and genes related to gut immunity that were significantly affected by FF treatment in the colon of pigs. The color represents a significant correlation $(P<0.05)$, and the intensity of the colors represents the degree of association. Red represents significant positive correlation and blue represents significantly negative correlation $(n=6)$.

study, we performed the study at an average initial body weight $(53.19 \pm 2.17$ vs. $54.60 \pm 1.62 \mathrm{~kg})$, and an interesting finding in this study was that FF meal greatly reduced the time to market (76 vs. 56 days). As compared with the UF group, the average daily weight gain and weight gain: food ratio in the FF group were significant increased $(P<0.05)$, which reflects improved growth performance and feed conversion efficiency, in accordance with the findings of previous studies (Canibe and Jensen, 2003). The fermentation process is believed to promote functional activities, such as antimicrobial and antioxidant activities, and increases the production of growth factors, hormones, and amino acids (Ng et al., 2011; Laskowska et al., 2017).

Proper function of the immune system is important for grower-finisher pigs. Immunoglobulins are immune-active molecules that play important roles in the humoral immune response (Kong et al., 2007). In the present study, the concentrations of IgG and IgM were significantly greater in the FF group $(P<0.05)$. The levels of IgG reflected immune status (Machado-Neto et al., 1987). IgM is associated with antiinflammation and a higher concentration reflects better immune status (Vaschetto et al., 2017). Our results were similar to those of Zhu et al. (2017), who reported that weaned piglets fed fermented soybean meal had higher serum concentrations of IgG and IgM, as compared with the controls. Some probiotic strains can be used as immunomodulators to enhance the concentrations of serum immunoglobulins (Vitini et al., 2000). Laskowska et al. (2017) found that dietary supplementation of Effective Microorganisms activated and enhanced the humoral and cell-mediated immune responses and protected against infection. Although high concentrations of immunoglobulins were observed, there was no intestinal inflammation, suggesting that the immunoglobulins were in the normal ranges. Moreover, FF meal decreased the concentrations of serum ALT and AST $(P>0.5)$. Reportedly, ALT is a liver-specific enzyme and ALT concentrations increased in response to acute liver injury (Robertson et al., 2016). Here, the lower levels of ALT and AST indicated that FF boosted overall health. In short, FF meal enhanced immune performance.

Toll-like receptors are the earliest discovered pattern recognition receptors and play critical roles in innate immunity (Newburg and Walker, 2007; Werling and Coffey, 2007), and TLR expression levels may indicate disease resistance in pigs (Uenishi and Shinkai, 2009; Cheng et al., 2015). Our data showed that the mRNA abundances of TLRs in the FF group were significantly greater than in the control group $(P<0.05)$. In this study, in the duodenum of FF-fed pigs, the mRNA abundances of PBD-1 and PR39 were significantly higher than that of the UF control pigs $(P<0.05)$. Meanwhile, the gene expression of $P B D-1$ in the colon was also higher than that of the UF meal pigs $(P<0.01)$. These results indicate that FF may benefits the pig gut immunity and in order to fully understand the regulating mechanism of FF on gut immunity, in vivo pathogenic challenge model is needed in further studies.

We then used a high-throughput sequencing method based on the 16S rRNA genes to demonstrate the effects of FF on the intestinal microbiota of grower-finisher pigs. In this study, FF had no effect on gut microbial community evenness (ace, Shannon $\mathrm{H}$ ) and richness (Simpson and Chao 1). Previous studies have shown that probiotic-supplemented FF decreased microbial diversity, which may be linked with improved resistance to gastrointestinal disorders (Ott et al., 2004; He et al., 2017). Similar to previous studies (Kim et al., 2012; Looft et al., 2012; 


\section{LC-MS(ESI-)}

A

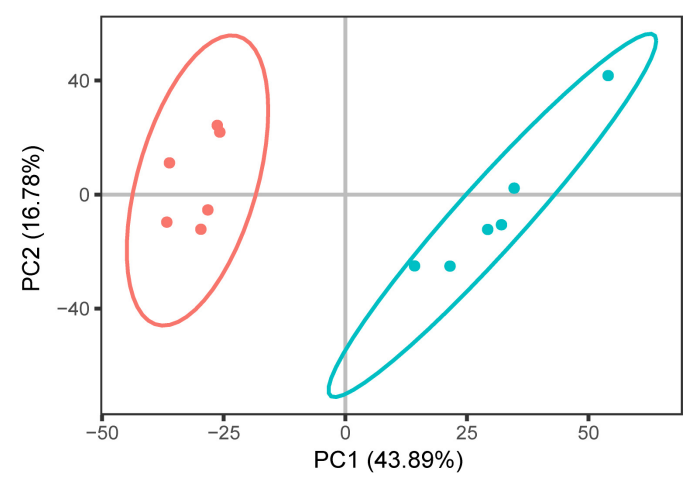

C

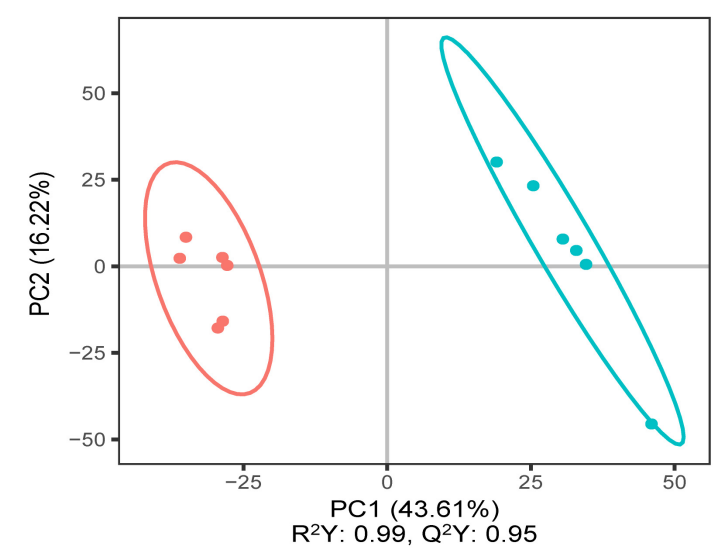

E

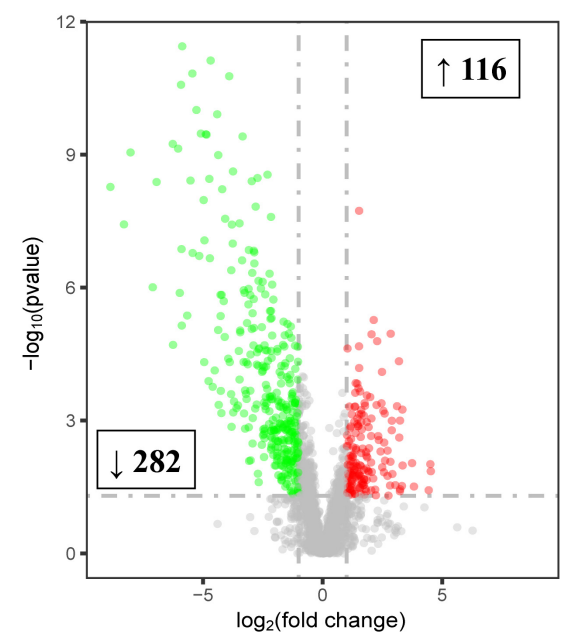

LC-MS(ESI+)

B

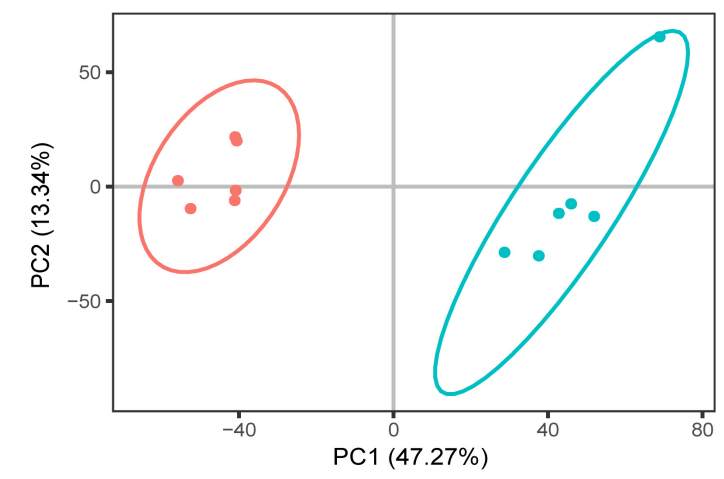

D

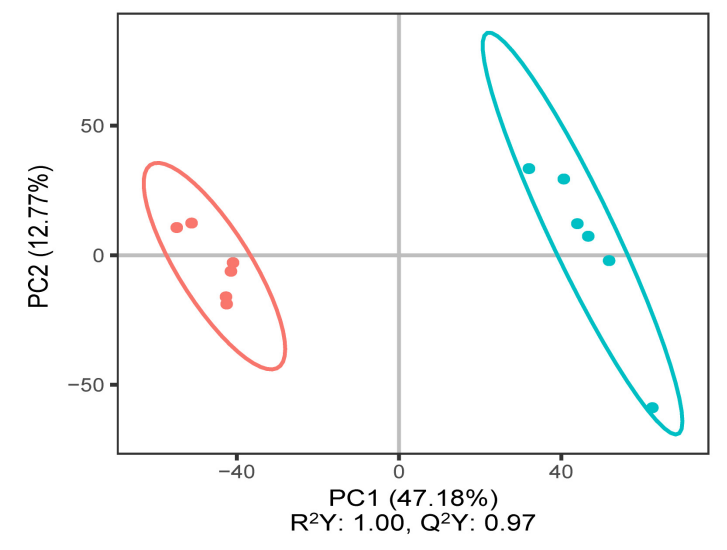

F

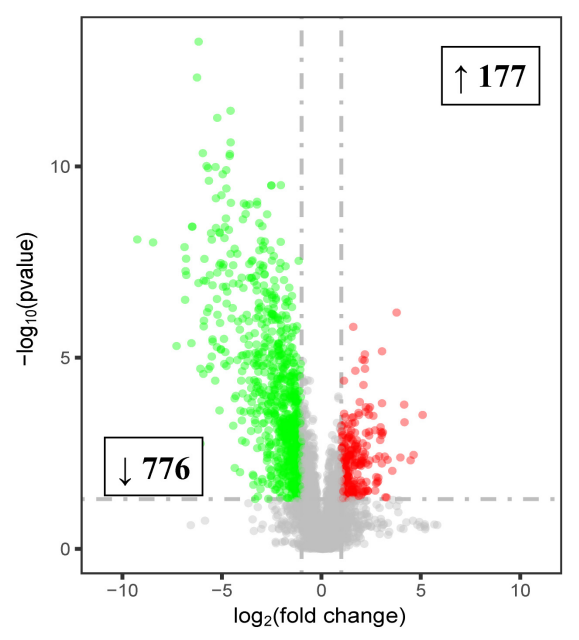

FIGURE 4 | Multivariate statistical analysis of untargeted metabolomics data obtained using the LC-MS/MS approach. PCA score plot of colonic metabolomics data for treatment (blue) and control (red) pigs obtained by (A) LC-MS (ESI-) and (B) LC-MS (ESI+) $(n=6)$. (C) PLS-DA score plot of colonic metabolomics data obtained by LC-MS (ESI-); $R^{2} Y=0.99 ; Q^{2}=0.95$. (D) PLS-DA score plot of colonic metabolomics data obtained by LC-MS (ESI+) data; $R^{2} Y=1.00 ; Q^{2}=0.97$. (E) Score plot of LC-MS (ESI-) data with 2,395 metabolite signals detected. (F) Score plot of LC-MS (ESI+) data with 5,708 metabolite signals detected. Red circles in volcano plots are model-separated metabolites following the conditions of VIP $>1$ and $\mid \mathrm{P}($ corr $) \mid \geq 0.5$ with $95 \%$ jack-knifed confidence intervals. Red or green rectangles indicate the numbers and tendency of metabolites to separate in the model when FF group pigs are compared with UF group. 


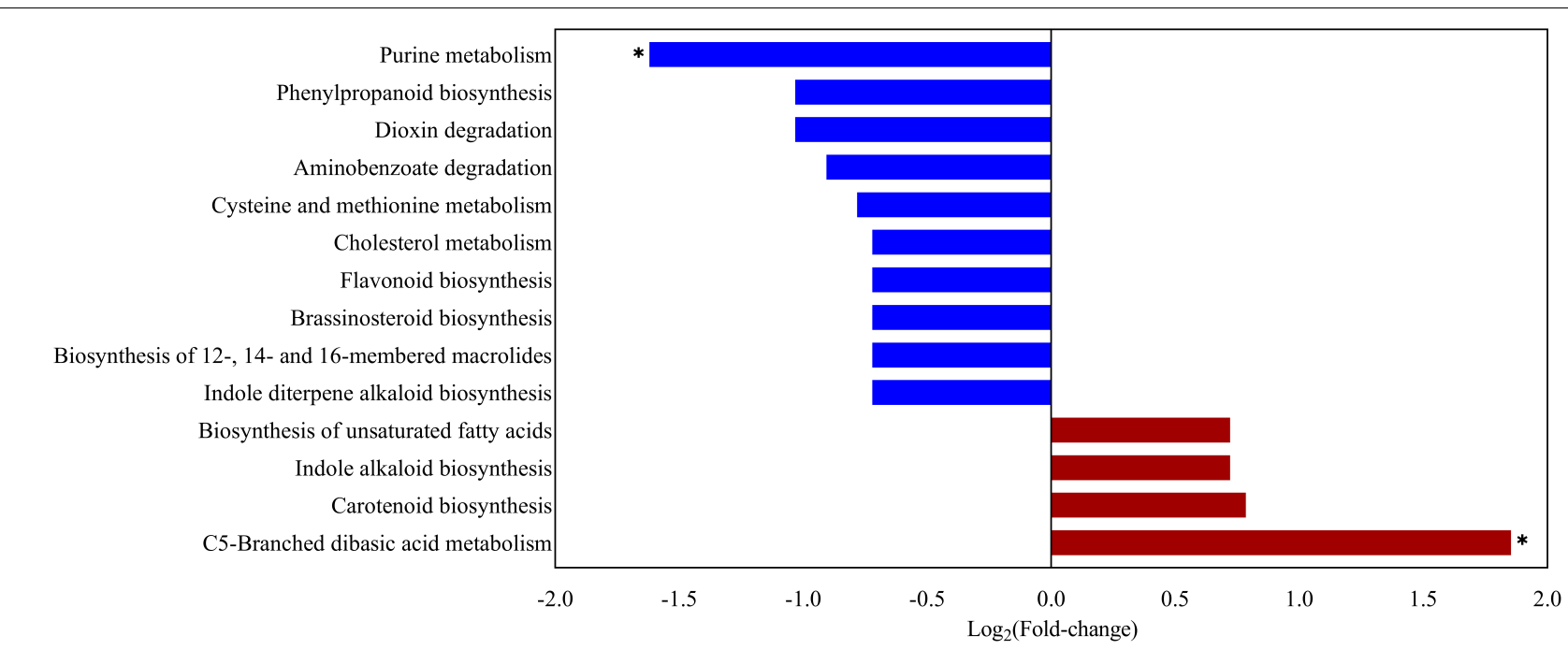

FIGURE 5 | KEGG pathway enrichment of the changed metabolites. Overview of metabolites that were enriched in pigs fed the FF meal compared to the UF meal. Length intensity indicates the $\log _{2}$-fold change in magnitude, using red for upregulation and blue for downregulation ( $\left.P<0.05\right)$.

Niu et al., 2015; Li et al., 2017), Firmicutes and Bacteroidetes were the most dominant phyla in the present study. Studies have shown that in obese animals, the ratio of Firmicutes to Bacteroides is increased (Ley et al., 2005, 2006b). In the present study, the ratio of the Firmicutes to Bacteroides was increased in both the duodenum and colon of the treatment group, which indicated that the use of FF changed the proportion of the microbiota and is beneficial to the weight gain of finisher pigs. In addition, a microbiome enriched in Firmicutes has been associated with an increased capacity for energy harvest and obesity (Ley et al., 2006a; Turnbaugh et al., 2006), and an increase of this phylum could therefore increase the amount of calories extracted from the diet. Compared with the control group, the relative abundance of Verrucomicrobia was significantly decreased in the FF group in the duodenum. Verrucomicrobia usually represents a minor population of intestinal microbiota in response to dietary shifts in mice (Pantoja-Feliciano et al., 2013). A relatively smaller proportion of Proteobacteria in the FF group was detected in our current study. It's reported that the members of the phylum Proteobacteria have a low abundance in the gut of healthy humans (Shin et al., 2015) and the increased levels of Proteobacteria may be indicative of a diseased state that commonly occurs during enteric infection or following perturbation of the microbiota (Singh et al., 2015). Moreover, FF significantly changed the gut microbiota composition, as indicated by decreased proportions, and significantly increased the proportions of Actinobacteria $(P<0.05)$. Meanwhile, we found there were five bacterial strains were significantly increased and 17 bacterial strains were significantly decreased in the colon in response to FF meal at the family level $(P<0.05)$; further studies are needed to investigate the roles of these gut bacteria in regulating the swine gut development.

The gut microbiota is important to host health and physiology status (Lalles, 2016). It is reported that microbes in the large intestine undertake more metabolism tasks
(Zhao et al., 2015). Using a KEGG pathway analysis, we found that C5-Branched dibasic acid metabolism was significantly upregulated whereas the purine metabolism was significantly downregulated $(P<0.05)$. Similarly, Gomez et al. (2017) measured the fecal microbiota and calculated the functional potential of the microbial communities in healthy and diarrheic calves. They found that C5-branched dibasic acid metabolism was enriched in healthy calves, suggesting that C5-branched dibasic acid metabolism is related to energy generation (Turnbaugh et al., 2006). The purine content of animal feed is a concern because excess intake of purines may increase the risk of hyperuricemia and gout (Zheng et al., 2018). It is necessary to conduct further research beyond the scope of the present study.

Fermented feed meal altered the metabolic functions and phenotypes of gut microbiota in pigs. The relative abundances of bacteria at the family levels were closely associated with the concentration of specific microbial metabolites in the colonic digesta. For instance, Lachnospiraceae is a bacterial family known to be abundant in the intestinal ecosystem (Sagheddu et al., 2016), and it is reported that Lachnospiraceae was positively correlated with carbohydrate metabolism (Morgan et al., 2012). Moreover, we found that Lachnospiraceae was also positively correlated with Sorbitan monooleate, N-Acetyl-L-aspartic acid, $\mathrm{N}$-Acetylcadaverine, and Mucronine B, whereas it negatively correlated with Caffeic acid phenethyl ester, Dodecanedioic acid, Torsemide, N-Acetyl-L-leucine, and Viloxazine. The mechanism underlying the dietary regulation of host phenotypes should be explored with a particular focus on metabolism and related receptors.

Further studies are needed to explore the benefits of FF benefits on the gut microbiota and health of pigs. To this end, fecal microbiota transplantation (FMT) may be an effective strategy. FMT refers to the process of transplantation of fecal bacteria from healthy individuals into a recipient (Drekonja et al., 2015). Growing evidence has shown that the host phenotypes, 


\section{A}

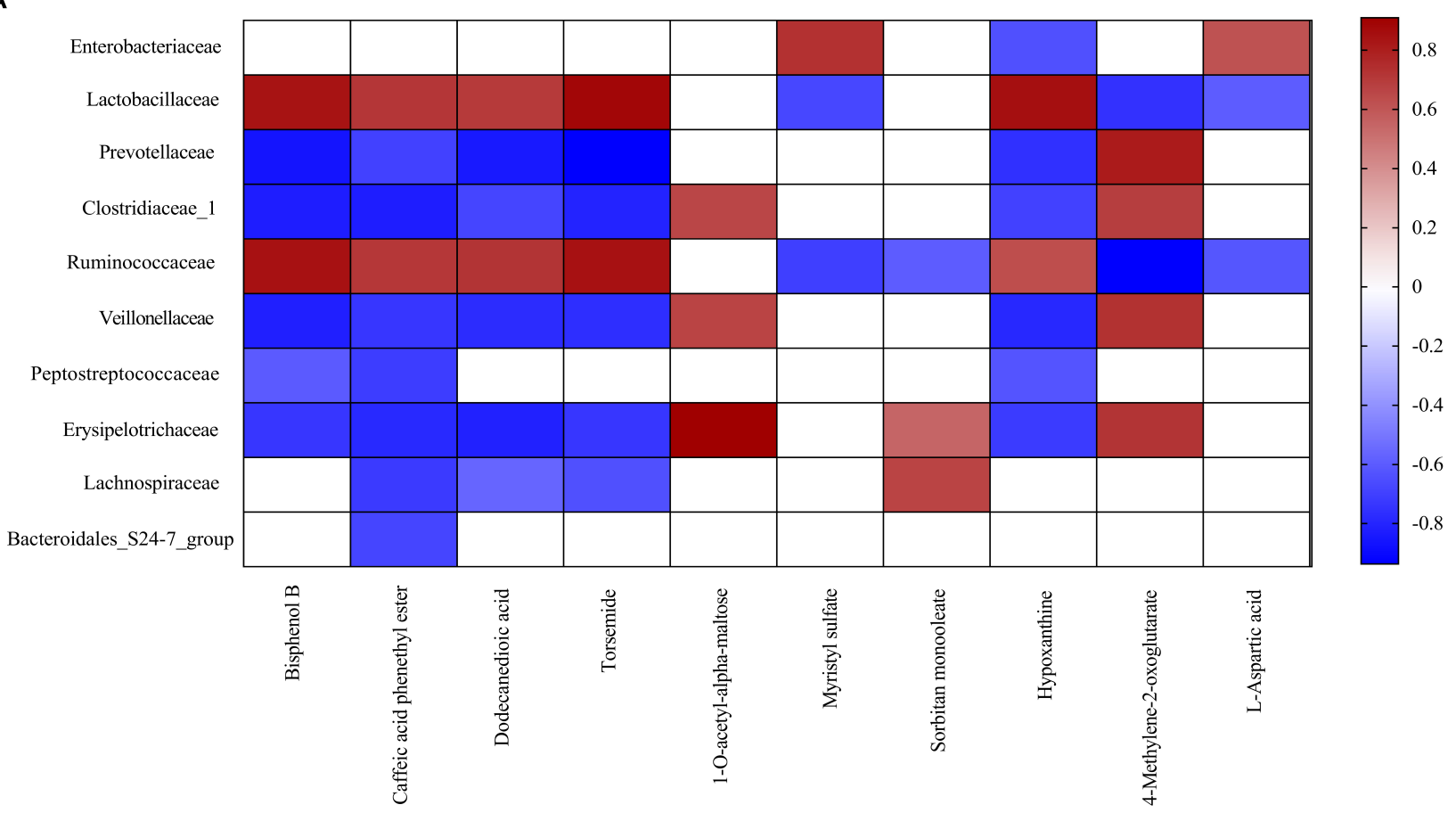

B

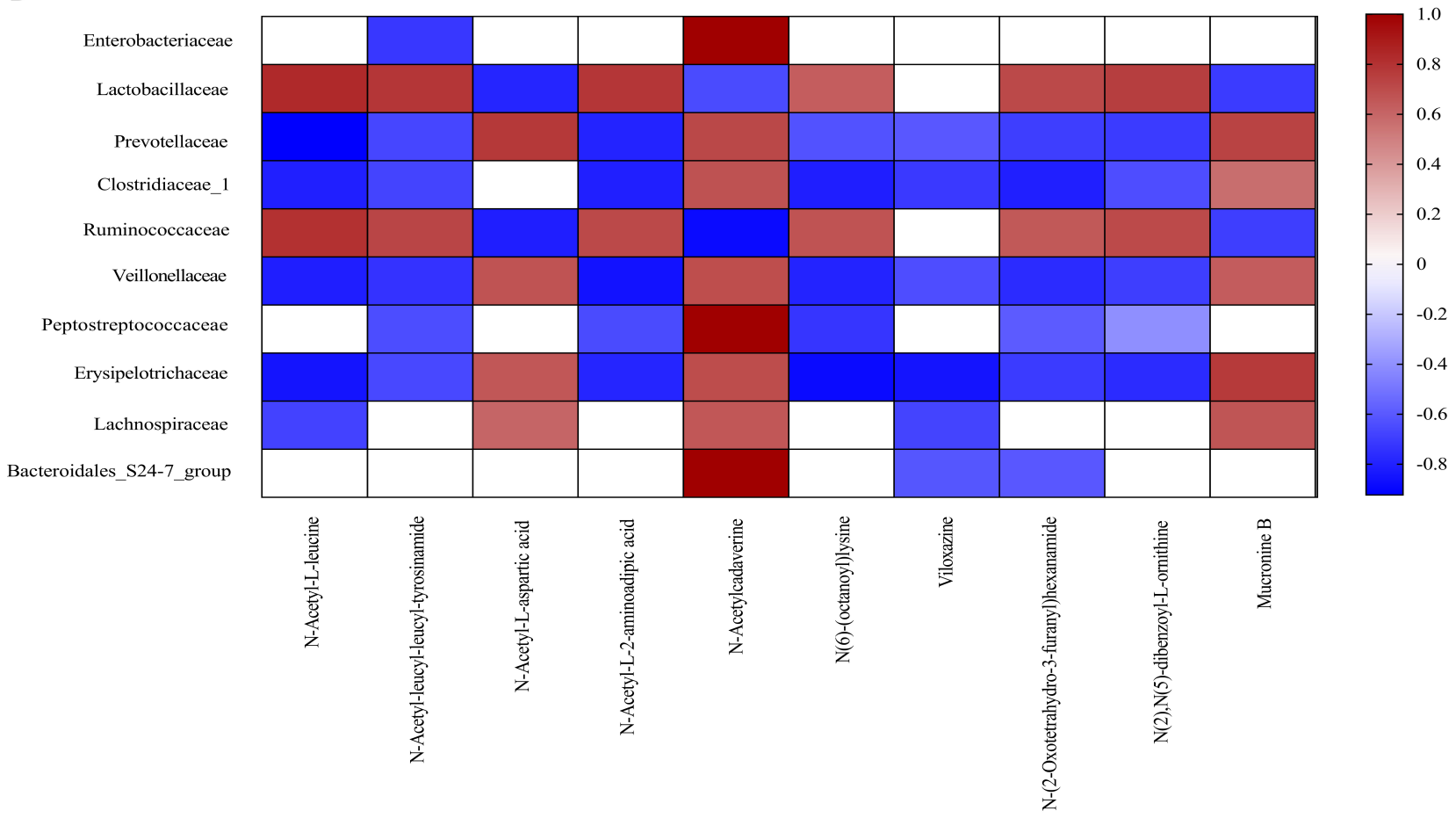

FIGURE 6 | Correlation analysis between colonic metabolites and colonic bacterial family-level taxa in pigs fed the FF meal compared to the UF meal. (A) LC-MS (ESI-). (B) LC-MS (ESI+). The color represents a significant correlation $(P<0.05)$, and the intensity of the colors represents the degree of association. Red represents significant positive correlation and blue represents significantly negative correlation $(n=6)$.

such as obesity (Lee et al., 2019), Clostridium difficile infection (Khoruts and Sadowsky, 2016), and anti-seizure (Olson et al., 2018) and anti-tumor immunity (Sivan et al., 2015), can be altered by FMT in mammals, demonstrating the critical roles of gut microbiota in host health. Thus, FMT also helped evaluate the potential links between intestinal microbiota and 
host phenotypes. Recently, $\mathrm{Hu}$ et al. (2018) proposed the standardized preparation for FMT for use in pig production and to identify host microbiota-derived bacteriocin targets to determine diarrhea resistance in early-weaned piglets (Hu et al., 2018). In the current study, we determined the FF could benefits the pig's gut microbiota; it would be interesting to explore the mechanism and we would like to focus on this in our next study.

\section{CONCLUSION}

In conclusion, we found that long-term consumption of FF meal increased the serum concentrations of IgG and IgM and changed the expression of genes related to gut immunity, which may be associated with alterations in the microbiota community and microbial metabolites and benefits to the pig's health. In addition, the results suggest that FF altered the microbial composition and modulated the metabolic pathway of microbial metabolism in pig colons. These alterations provide an alternative strategy for improving the intestinal health of pigs.

\section{DATA AVAILABILITY STATEMENT}

The datasets generated for this study can be found in NCBI, No. PRJNA524989.

\section{ETHICS STATEMENT}

The use of animals and the performance of all experimental protocols were approved by the Northwest A\&F University Animal Welfare Committee (Yangling, Shaanxi Province, China).

\section{REFERENCES}

Ananthakrishnan, A. N. (2015). Epidemiology and risk factors for IBD. Nat. Rev. Gastroenterol. Hepatol. 12, 205-217. doi: 10.1038/nrgastro.2015.34

Borling Welin, J., Lyberg, K., Passoth, V., and Olstorpe, M. (2015). Combined moist airtight storage and feed fermentation of barley by the yeast Wickerhamomyces anomalus and a lactic acid bacteria consortium. Front. Plant. Sci. 6:270. doi: $10.3389 /$ fpls.2015.00270

Canibe, N., and Jensen, B. B. (2003). Fermented and nonfermented liquid feed to growing pigs: effect on aspects of gastrointestinal ecology and growth performance. J. Anim. Sci. 81, 2019-2031. doi: 10.2527/2003.8182019x

Caporaso, J. G., Kuczynski, J., Stombaugh, J., Bittinger, K., Bushman, F. D., Costello, E. K., et al. (2010). QIIME allows analysis of high-throughput community sequencing data. Nat. Methods 7, 335-336. doi: 10.1038/nmeth.f. 303

Cheng, C., Sun, W. K., Liu, R., Wang, R. M., Chen, Y. H., Wang, Y., et al. (2015). Comparison of gene expression of Toll-like receptors and antimicrobial peptides in immune organs and tissues between Yorkshire and Tibetan pigs. Anim. Genet. 46, 272-279. doi: 10.1111/age.12286

DeSantis, T. Z., Hugenholtz, P., Larsen, N., Rojas, M., Brodie, E. L., Keller, K., et al. (2006). Greengenes, a chimera-checked 16S rRNA gene database and workbench compatible with ARB. Appl. Environ. Microbiol. 72, 5069-5072. doi: 10.1128/aem.03006-05

Dibner, J. J., and Richards, J. D. (2005). Antibiotic growth promoters in agriculture: history and mode of action. Poul. Sci. 84, 634-643. doi: 10.1093/ps/84.4.634

Dowarah, R., Verma, A. K., Agarwal, N., Patel, B. H. M., and Singh, P. (2017). Effect of swine based probiotic on performance, diarrhoea scores, intestinal
The processing of animal experiments and sample collection strictly followed the relevant guidelines.

\section{AUTHOR CONTRIBUTIONS}

$\mathrm{XS}$ and JL designed the study, and wrote and revised the manuscript. JL, XZ, HC, QH, BX, and LF helped took samples and performed the experiments and analyses. JL, YL, XL, JH, GY, and XS edited the manuscript. All authors reviewed the final manuscript.

\section{FUNDING}

This study was supported by the Agricultural Special Fund Project in Shaanxi Province (NYKJ-2018-YL01) and the Technical Innovation Guidance Special (Foundation) Project of Shaanxi Province (2017ZKC07-114).

\section{ACKNOWLEDGMENTS}

We thank others in Li's team and Shi's team for their excellent support during this experiment.

\section{SUPPLEMENTARY MATERIAL}

The Supplementary Material for this article can be found online at: https://www.frontiersin.org/articles/10.3389/fmicb. 2019.02620/full\#supplementary-material

microbiota and gut health of grower-finisher crossbred pigs. Livestock Sci. 195, 74-79. doi: 10.1016/j.livsci.2016.11.006

Drekonja, D., Reich, J., Gezahegn, S., Greer, N., Shaukat, A., MacDonald, R., et al. (2015). Fecal microbiota transplantation for clostridium difficile infection: a systematic review. Ann. Intern. Med. 162, 630-638. doi: 10.7326/M142693

Edgar, R. C. (2004). MUSCLE: multiple sequence alignment with high accuracy and high throughput. Nucleic Acids Res. 32, 1792-1797. doi: 10.1093/nar/gkh340

Edgar, R. C. (2013). UPARSE: highly accurate OTU sequences from microbial amplicon reads. Nature Methods 10:996. doi: 10.1038/nmeth.2604

Edgar, R. C., Haas, B. J., Clemente, J. C., Quince, C., and Knight, R. (2011). UCHIME improves sensitivity and speed of chimera detection. Bioinformatics 27, 2194-2200. doi: 10.1093/bioinformatics/btr381

El Aidy, S., Merrifield, C. A., Derrien, M., van Baarlen, P., Hooiveld, G., Levenez, F., et al. (2013). The gut microbiota elicits a profound metabolic reorientation in the mouse jejunal mucosa during conventionalisation. Gut 62, 1306-1314. doi: 10.1136/gutjnl-2011-301955

Frederick, B. R., van Heugten, E., and See, M. T. (2006). Effects of pig age at market weight and magnesium supplementation through drinking water on pork quality. J. Anim. Sci. 84, 1512-1519. doi: 10.2527/2006.846 $1512 \mathrm{x}$

Frese, S. A., Parker, K., Calvert, C. C., and Mills, D. A. (2015). Diet shapes the gut microbiome of pigs during nursing and weaning. Microbiome 3:28. doi: 10.1186/s40168-015-0091-98

Gomez, D. E., Arroyo, L. G., Costa, M. C., Viel, L., and Weese, J. S. (2017). Characterization of the fecal bacterial microbiota of healthy and diarrheic dairy calves. J. Vet. Intern. Med. 31, 928-939. doi: 10.1111/jvim.14695 
Gu, M., Zhao, P., Zhang, S., Fan, S., Yang, L., Tong, Q., et al. (2019). Betulinic acid alleviates endoplasmic reticulum stress-mediated nonalcoholic fatty liver disease through activation of farnesoid $\mathrm{X}$ receptors in mice. Br. J. Pharmacol. 176, 847-863. doi: 10.1111/bph.14570

Guevarra, R. B., Lee, J. H., Lee, S. H., Seok, M. J., Kim, D. W., Kang, B. N., et al. (2019). Piglet gut microbial shifts early in life: causes and effects. J. Anim. Sci. Biotechnol. 10:1. doi: 10.1186/s40104-018-0308-303

Haas, B. J., Gevers, D., Earl, A. M., Feldgarden, M., Ward, D. V., Giannoukos, G., et al. (2011). Chimeric 16S rRNA sequence formation and detection in Sanger and 454-pyrosequenced PCR amplicons. Genom. Res. 21, 494-504. doi: $10.1101 /$ gr.112730.110

He, Y., Mao, C., Wen, H., Chen, Z., Lai, T., Li, L., et al. (2017). Influence of ad libitum feeding of piglets with bacillus subtilis fermented liquid feed on gut flora. Luminal Contents Health. Sci. Rep. 7:44553. doi: 10.1038/srep44553

Hu, J., Chen, L., Tang, Y., Xie, C., Xu, B., Shi, M., et al. (2018). Standardized preparation for fecal microbiota transplantation in pigs. Front. Microbiol. 9:1328. doi: 10.3389/fmicb.2018.01328

Isaacson, R., and Kim, H. B. (2012). The intestinal microbiome of the pig. Anim. Health Res. Rev. 13, 100-109. doi: 10.1017/S1466252312000084

Jeong, J. S., Park, J. W., Lee, S. I., and Kim, I. H. (2016). Apparent ileal digestibility of nutrients and amino acids in soybean meal, fish meal, spray-dried plasma protein and fermented soybean meal to weaned pigs. Anim. Sci. J. 87, 697-702. doi: $10.1111 /$ asj. 12483

Ji, Y., Guo, Q., Yin, Y., Blachier, F., and Kong, X. (2018). Dietary proline supplementation alters colonic luminal microbiota and bacterial metabolite composition between days 45 and 70 of pregnancy in Huanjiang mini-pigs. J. Anim. Sci. Biotechnol. 9:18. doi: 10.1186/s40104-018-0233-235

Jin, W., Ye, H., Zhao, J.-Z., Zhou, Z. J., and Huan, F. (2017). Consuming fermented distillers' dried grains with solubles (DDGS) feed reveals a shift in the faecal microbiota of growing and fattening pigs using 454 pyrosequencing. J. Integr. Agric 16, 900-910. doi: 10.1016/s2095-3119(16)61523-x

Khoruts, A., and Sadowsky, M. J. (2016). Understanding the mechanisms of faecal microbiota transplantation. Nat. Rev. Gastroenterol. Hepatol. 13, 508-516. doi: 10.1038/nrgastro.2016.98

Kim, H. B., Borewicz, K., White, B. A., Singer, R. S., Sreevatsan, S., Tu, Z. J., et al. (2012). Microbial shifts in the swine distal gut in response to the treatment with antimicrobial growth promoter, tylosin. Proc. Natl. Acad. Sci. U.S.A. 109, 15485-15490. doi: 10.1073/pnas.1205147109

Kim, Y., Kim, S., Weaver, M., and Lee, C. (2005). Increasing the pig market weight: world trends, expected consequences and practical considerations. Asian Aust. J. Anim. Sci. 18, 590-600. doi: 10.5713/ajas.2005.590

Kong, X., Yin, Y., Wu, G., Liu, H., Yin, F., Li, T., et al. (2007). Dietary Supplementation with acanthopanax senticosus extract modulates cellular and humoral immunity in weaned piglets. Asian Aust. J. Anim. Sci. 20, 1453-1461. doi: 10.5713/ajas.2007.1453

Lalles, J. P. (2016). Microbiota-host interplay at the gut epithelial level, health and nutrition. J. Anim. Sci. Biotechnol. 7:66. doi: 10.1186/s40104-016-0123-127

Laskowska, E., Jarosz, L., and Gradzki, Z. (2017). The effect of feed supplementation with effective microorganisms (EM) on pro- and antiinflammatory cytokine concentrations in pigs. Res. Vet. Sci. 115, 244-249. doi: 10.1016/j.rvsc.2017.03.008

Lee, P., Yacyshyn, B. R., and Yacyshyn, M. B. (2019). Gut microbiota and obesity: an opportunity to alter obesity through faecal microbiota transplant (FMT). Diabetes Obes. Metab. 21, 479-490. doi: 10.1111/dom.13561

Ley, R. E., Backhed, F., Turnbaugh, P., Lozupone, C. A., Knight, R. D., and Gordon, J. I. (2005). Obesity alters gut microbial ecology. Proc. Natl. Acad. Sci. U.S.A. 102, 11070-11075. doi: 10.1073/pnas.0504978102

Ley, R. E., Peterson, D. A., and Gordon, J. I. (2006a). Ecological and evolutionary forces shaping microbial diversity in the human intestine. Cell 124, 837-848. doi: 10.1016/j.cell.2006.02.017

Ley, R. E., Turnbaugh, P. J., Klein, S., and Gordon, J. I. (2006b). Microbial ecology: human gut microbes associated with obesity. Nature 444, 1022-1023. doi: 10 . 1038/4441022a

Li, P., Niu, Q., Wei, Q., Zhang, Y., Ma, X., Kim, S. W., et al. (2017). Microbial shifts in the porcine distal gut in response to diets supplemented with Enterococcus Faecalis as alternatives to antibiotics. Sci. Rep. 7:41395. doi: 10.1038/srep 41395
Looft, T., Johnson, T. A., Allen, H. K., Bayles, D. O., Alt, D. P., Stedtfeld, R. D., et al. (2012). In-feed antibiotic effects on the swine intestinal microbiome. Proc. Natl. Acad. Sci. U.S.A. 109, 1691-1696. doi: 10.1073/pnas.1120238109

Machado-Neto, R., Graves, C. N., and Curtis, S. E. (1987). Immunoglobulins in piglets from sows heat-stressed prepartum. J. Anim. Sci. 65, 445-455. doi: $10.2527 /$ jas $1987.652445 \mathrm{x}$

Magoc, T., and Salzberg, S. L. (2011). FLASH: fast length adjustment of short reads to improve genome assemblies. Bioinformatics 27, 2957-2963. doi: 10.1093/ bioinformatics/btr507

Markowiak, P., and Slizewska, K. (2018). The role of probiotics, prebiotics and synbiotics in animal nutrition. Gut. Pathog. 10:21. doi: 10.1186/s13099-0180250- 250

Missotten, J. A., Michiels, J., Degroote, J., and De Smet, S. (2015). Fermented liquid feed for pigs: an ancient technique for the future. J. Anim. Sci. Biotechnol. 6:4. doi: 10.1186/2049-1891-6-4

Morgan, X. C., Tickle, T. L., Sokol, H., Gevers, D., Devaney, K. L., Ward, D. V., et al. (2012). Dysfunction of the intestinal microbiome in inflammatory bowel disease and treatment. Genom. Biol. 13:R79. doi: 10.1186/gb-2012-13-9-r79

Newburg, D. S., and Walker, W. A. (2007). Protection of the neonate by the innate immune system of developing gut and of human milk. Pediatr. Res. 61, 2-8. doi: 10.1203/01.pdr.0000250274.68571.18

Ng, C. C., Wang, C. Y., Wang, Y. P., Tzeng, W. S., and Shyu, Y. T. (2011). Lactic acid bacterial fermentation on the production of functional antioxidant herbal Anoectochilus formosanus Hayata. J. Biosci. Bioeng. 111, 289-293. doi: 10.1016/ j.jbiosc.2010.11.011

Niu, Q., Li, P., Hao, S., Zhang, Y., Kim, S. W., Li, H., et al. (2015). Dynamic distribution of the gut microbiota and the relationship with apparent crude fiber digestibility and growth stages in pigs. Sci. Rep. 5:9938. doi: 10.1038/srep09938

NRC, (2012). NutrientRrequirements of Swine. Models for Estimating Nutrient Requirements of Pigs-Case Studies, 11th Edn. Washington DC: National Research Council, National.

Olson, C. A., Vuong, H. E., Yano, J. M., Liang, Q. Y., Nusbaum, D. J., and Hsiao, E. Y. (2018). The gut microbiota mediates the anti-seizure effects of the ketogenic diet. Cell 172:e1713. doi: 10.1016/j.cell.2018.04.027

Ott, S. J., Musfeldt, M., Wenderoth, D. F., Hampe, J., Brant, O., Folsch, U. R., et al. (2004). Reduction in diversity of the colonic mucosa associated bacterial microflora in patients with active inflammatory bowel disease. Gut 53, 685-693. doi: 10.1136/gut.2003.025403

Pantoja-Feliciano, I. G., Clemente, J. C., Costello, E. K., Perez, M. E., Blaser, M. J., Knight, R., et al. (2013). Biphasic assembly of the murine intestinal microbiota during early development. ISME J. 7, 1112-1115. doi: 10.1038/ismej. 2013.15

Richards, J., Gong, J., and De Lange, C. (2005). The gastrointestinal microbiota and its role in monogastric nutrition and health with an emphasis on pigs: Current understanding, possible modulations, and new technologies for ecological studies. Can. J. Anim. Sci. 85, 421-435. doi: 10.4141/a05-049

Robertson, F. P., Bessell, P. R., Diaz-Nieto, R., Thomas, N., Rolando, N., Fuller, B., et al. (2016). High serum Aspartate transaminase levels on day 3 postliver transplantation correlates with graft and patient survival and would be a valid surrogate for outcome in liver transplantation clinical trials. Transpl. Int. 29, 323-330. doi: 10.1111/tri.12723

Sagheddu, V., Patrone, V., Miragoli, F., Puglisi, E., and Morelli, L. (2016). Infant early gut colonization by lachnospiraceae: high frequency of ruminococcus gnavus. Front. Pediatr. 4:57. doi: 10.3389/fped.2016.00057

Shi, C., Zhang, Y., Lu, Z., and Wang, Y. (2017). Solid-state fermentation of cornsoybean meal mixed feed with Bacillus subtilis and Enterococcus faecium for degrading antinutritional factors and enhancing nutritional value. J. Anim. Sci. Biotechnol. 8:50. doi: 10.1186/s40104-017-0184-182

Shin, N. R., Whon, T. W., and Bae, J. W. (2015). Proteobacteria: microbial signature of dysbiosis in gut microbiota. Trends Biotechnol. 33, 496-503. doi: 10.1016/j. tibtech.2015.06.011

Sieuwerts, S., de Bok, F. A., Mols, E., de vos, W. M., and Vlieg, J. E. (2008). A simple and fast method for determining colony forming units. Lett. Appl. Microbiol. 47, 275-278. doi: 10.1111/j.1472-765X.2008.02417.x

Singh, P., Teal, T. K., Marsh, T. L., Tiedje, J. M., Mosci, R., Jernigan, K., et al. (2015). Intestinal microbial communities associated with acute enteric infections and disease recovery. Microbiome 3:45. doi: 10.1186/s40168-015-0109-102 
Sivan, A., Corrales, L., Hubert, N., Williams, J. B., Aquino-Michaels, K., Earley, Z. M., et al. (2015). Commensal Bifidobacterium promotes antitumor immunity and facilitates anti-PD-L1 efficacy. Science 350, 1084-1089. doi: 10.1126/ science.aac 4255

Stanley, D., Hughes, R. J., Geier, M. S., and Moore, R. J. (2016). Bacteria within the gastrointestinal tract microbiota correlated with improved growth and feed conversion: challenges presented for the identification of performance enhancing probiotic Bacteria. Front. Microbiol. 7:187. doi: 10.3389/fmicb.2016. 00187

Thacker, P. A. (2013). Alternatives to antibiotics as growth promoters for use in swine production: a review. J. Anim. Sci. Biotechnol. 4:35. doi: 10.1186/20491891-4-35

Tian, X. Y., and Zhang, C. S. (2017). Illumina-based analysis of endophytic and rhizosphere bacterial diversity of the coastal halophyte Messerschmidia sibirica. Front. Microbiol. 8:2288. doi: 10.3389/fmicb.2017.02288

Tremaroli, V., and Backhed, F. (2012). Functional interactions between the gut microbiota and host metabolism. Nature 489, 242-249. doi: 10.1038/ nature 11552

Turnbaugh, P. J., Ley, R. E., Mahowald, M. A., Magrini, V., Mardis, E. R., and Gordon, J. I. (2006). An obesity-associated gut microbiome with increased capacity for energy harvest. Nature 444, 1027-1031. doi: 10.1038/nature05414

Uenishi, H., and Shinkai, H. (2009). Porcine toll-like receptors: the front line of pathogen monitoring and possible implications for disease resistance. Dev. Comp. Immunol. 33, 353-361. doi: 10.1016/j.dci.2008.06.001

van der Fels-Klerx, H. J., Puister-Jansen, L. F., van Asselt, E. D., and Burgers, S. L. (2011). Farm factors associated with the use of antibiotics in pig production. J. Anim. Sci. 89, 1922-1929. doi: 10.2527/jas.2010-3046

Vaschetto, R., Clemente, N., Pagni, A., Esposito, T., Longhini, F., Mercalli, F., et al. (2017). A double blind randomized experimental study on the use of IgM-enriched polyclonal immunoglobulins in an animal model of pneumonia developing shock. Immunobiology 222, 1074-1080. doi: 10.1016/j.imbio.2017. 09.002

Vermeer, H., de Greef, K., and Houwers, H. (2014). Space allowance and pen size affect welfare indicators and performance of growing pigs under Comfort Class conditions. Livestock Sci. 159, 79-86. doi: 10.1016/j.livsci.2013.10.021

Verstegen, M. W., and Williams, B. A. (2002). Alternatives to the use of antibiotics as growth promoters for monogastric animals. Anim. Biotechnol. 13, 113-127. doi: 10.1081/ABIO-120005774

Vitini, E., Alvarez, S., Medina, M., Medici, M., de Budeguer, M. V., and Perdigon, G. (2000). Gut mucosal immunostimulation by lactic acid bacteria. Biocell 24, 223-232.

Wang, C., Shi, C., Zhang, Y., Song, D., Lu, Z., and Wang, Y. (2018). Microbiota in fermented feed and swine gut. Appl. Microbiol. Biotechnol. 102, 2941-2948. doi: $10.1007 /$ s00253-018-8829-8824
Wang, J., Li, D., Wang, P., Hu, X., and Chen, F. (2019). Ginger prevents obesity through regulation of energy metabolism and activation of browning in high-fat diet-induced obese mice. J. Nutr. Biochem. 70, 105-115. doi: 10.1016/j.jnutbio. 2019.05.001

Wang, P., Zhou, Q., Feng, J., He, J., Lou, Y., and Zhu, J. (2019). Effect of dietary fermented soybean meal on growth, intestinal morphology and microbiota in juvenile large yellow croaker, Larimichthys crocea. Aquac. Res. 50, 748-757. doi: 10.1111/are.13929

Wang, W., Hu, H., Zijlstra, R. T., Zheng, J., and Ganzle, M. G. (2019). Metagenomic reconstructions of gut microbial metabolism in weanling pigs. Microbiome 7:48. doi: 10.1186/s40168-019-0662-661

Wang, Q., Garrity, G. M., Tiedje, J. M., and Cole, J. R. (2007). Naive Bayesian classifier for rapid assignment of rRNA sequences into the new bacterial taxonomy. Appl. Environ. Microbiol. 73, 5261-5267. doi: 10.1128/aem. 00062-07

Werling, D., and Coffey, T. J. (2007). Pattern recognition receptors in companion and farm animals - the key to unlocking the door to animal disease? Vet. J. 174, 240-251. doi: 10.1016/j.tvjl.2006. 10.010

Zhao, W., Wang, Y., Liu, S., Huang, J., Zhai, Z., He, C., et al. (2015). The dynamic distribution of porcine microbiota across different ages and gastrointestinal tract segments. PLoS One 10:e0117441. doi: 10.1371/journal.pone.011 7441

Zheng, M., Huang, Y., Ji, J., Xiao, S., Ma, J., and Huang, L. (2018). Effects of breeds, tissues and genders on purine contents in pork and the relationships between purine content and other meat quality traits. Meat. Sci. 143, 81-86. doi: 10.1016/j.meatsci.2018.04.022

Zhu, J., Gao, M., Zhang, R., Sun, Z., Wang, C., Yang, F., et al. (2017). Effects of soybean meal fermented by L. plantarum, B. subtilis and S. cerevisieae on growth, immune function and intestinal morphology in weaned piglets. Microb. Cell Fact. 16:191. doi: 10.1186/s12934-017-08 09-803

Conflict of Interest: The authors declare that the research was conducted in the absence of any commercial or financial relationships that could be construed as a potential conflict of interest.

Copyright (c) 2019 Lu, Zhang, Liu, Cao, Han, Xie, Fan, Li, Hu, Yang and Shi. This is an open-access article distributed under the terms of the Creative Commons Attribution License (CC BY). The use, distribution or reproduction in other forums is permitted, provided the original author(s) and the copyright owner(s) are credited and that the original publication in this journal is cited, in accordance with accepted academic practice. No use, distribution or reproduction is permitted which does not comply with these terms. 